\title{
Numerator polynomials of the Riordan matrices
}

\author{
E. Burlachenko
}

\begin{abstract}
Riordan matrices are infinite lower triangular matrices corresponding to the certain operators in the space of formal power series. Generalized Euler polynomials $g_{n}(x)=(1-x)^{n+1} \sum_{m=0}^{\infty} p_{n}(m) x^{m}$, where $p_{n}(m)$ is the polynomial of degree $\leq n$, are the numerator polynomials of the generating functions of diagonals of the ordinary Riordan matrices. Generalized Narayana polynomials $h_{n}(x)=$ $(1-x)^{2 n+1} \sum_{m=0}^{\infty}(m+1) \ldots(m+n) p_{n}(m) x^{m}$ are the numerator polynomials of the generating functions of diagonals of the exponential Riordan matrices. In paper, the properties of these two types of numerator polynomials and the constructive relationships between them are considered. Separate attention is paid to the numerator polynomials of Riordan matrices associated with the family of series ${ }_{(\beta)} a(x)=a\left(x_{(\beta)} a^{\beta}(x)\right)$.
\end{abstract}

\section{Introduction}

Transformations in the space of formal power series over the field of real or complex numbers and the corresponding matrices are the subject of our study. We associate the rows and columns of matrices with the generating functions of their elements, i.e., formal power series. Thus, the expression $A a(x)=b(x)$ means that the column vector multiplied by the matrix $A$ has the generating function $a(x)=\sum_{n=0}^{\infty} a_{n} x^{n}$, resultant column vector has the generating function $b(x)=\sum_{n=0}^{\infty} b_{n} x^{n}$. $n$th coefficient of the series $a(x), n$th row, $n$th descending diagonal and $n$th column of the matrix $A$ will be denoted respectively by

$$
\left[x^{n}\right] a(x), \quad[n, \rightarrow] A, \quad[n, \searrow] A, \quad A x^{n} .
$$

Matrix $(f(x), g(x))$, nth column of which has the generating function $f(x) g^{n}(x)$, $g_{0}=0$, is called Riordan matrix (Riordan array) [1] - [3]. It is the product of two matrices that correspond to the operators of multiplication and composition of series:

$$
\begin{gathered}
(f(x), g(x))=(f(x), x)(1, g(x)), \\
(f(x), x) a(x)=f(x) a(x), \quad(1, g(x)) a(x)=a(g(x)), \\
(f(x), g(x))(b(x), a(x))=(f(x) b(g(x)), a(g(x))) .
\end{gathered}
$$

If $f_{0} \neq 0, g_{1} \neq 0$, matrix $(f(x), g(x))$ is called proper. Proper Riordan matrices form a group, called the Riordan group.

Matrices

$$
\left|e^{x}\right|^{-1}(f(x), g(x))\left|e^{x}\right|=(f(x), g(x))_{E},
$$

where $\left|e^{x}\right|$ is the diagonal matrix: $\left|e^{x}\right| x^{n}=x^{n} / n$ ! , are called exponential Riordan matrices [4], [5]. Denote $[n, \rightarrow](f(x), g(x))_{E}=s_{n}(x)$. Then

$$
(f(x), g(x))_{E}(1-\varphi x)^{-1}=\left|e^{x}\right|^{-1}(f(x), g(x)) e^{\varphi x}=\left|e^{x}\right|^{-1} f(x) \exp (\varphi g(x)),
$$

or

$$
\sum_{n=0}^{\infty} \frac{s_{n}(\varphi)}{n !} x^{n}=f(x) \exp (\varphi g(x))
$$


If $f_{0} \neq 0, g_{1} \neq 0$, sequence of polynomials $s_{n}(x)$ is called Sheffer sequence, and in the case $f(x)=1$ binomial sequence. Properties of the Sheffer sequences are subject of study of the umbral calculus [6]. Matrix

$$
P=\left(\frac{1}{1-x}, \frac{x}{1-x}\right)=\left(e^{x}, x\right)_{E}=\left(\begin{array}{ccccc}
1 & 0 & 0 & 0 & \cdots \\
1 & 1 & 0 & 0 & \cdots \\
1 & 2 & 1 & 0 & \cdots \\
1 & 3 & 3 & 1 & \cdots \\
\vdots & \vdots & \vdots & \vdots & \ddots
\end{array}\right)
$$

is called Pascal matrix. Power of the Pascal matrix is defined by

$$
P^{\varphi}=\left(\frac{1}{1-\varphi x}, \frac{x}{1-\varphi x}\right)=\left(e^{\varphi x}, x\right)_{E} .
$$

Along with the lower triangular Riordan matrices, we will consider the "square" matrices $(b(x), a(x)), b_{0} \neq 0, a_{0}=1$, whose $n$th column has the generating function $b(x) a^{n}(x)$. Upper triangular matrix $(1,1+x)$, transposed to the Pascal matrix and coinciding with the matrix of shift operator, also belongs to this type of matrices:

$$
(1,1+x)=P^{T}=E=\left(\begin{array}{ccccc}
1 & 1 & 1 & 1 & \cdots \\
0 & 1 & 2 & 3 & \cdots \\
0 & 0 & 1 & 3 & \cdots \\
0 & 0 & 0 & 1 & \cdots \\
\vdots & \vdots & \vdots & \vdots & \ddots
\end{array}\right)
$$

Matrix $(b(x), a(x))$ can be multiplied from the right by the matrix with the finite columns and from the left by the matrix with the finite rows. Since $[n, \rightarrow](b(x), a(x))=$ $=[n, \searrow](b(x), x a(x))$, then the matrix $(b(x), a(x))$ is a useful tool for studying the matrix $(b(x), x a(x))$. Denote

$$
[n, \rightarrow](b(x), a(x)-1)=w_{n}(x)=\sum_{m=0}^{n} w_{m} x^{m}
$$

Since

$$
(b(x), a(x))=(b(x), a(x)-1)(1,1+x), \quad[n, \rightarrow](1,1+x)=\frac{x^{n}}{(1-x)^{n+1}},
$$

then

$$
[n, \rightarrow](b(x), a(x))=\sum_{m=0}^{n} \frac{w_{m} x^{m}}{(1-x)^{m+1}}=\frac{\sum_{m=0}^{n} w_{m} x^{m}(1-x)^{n-m}}{(1-x)^{n+1}}=\frac{g_{n}(x)}{(1-x)^{n+1}} .
$$

If $b(x)=1, a(x)=e^{x}$, then $g_{n}(x)=A_{n}(x) / n$ !, where $A_{n}(x)$ are the Euler polynomials:

$$
\frac{A_{n}(x)}{(1-x)^{n+1}}=\sum_{m=0}^{\infty} m^{n} x^{m}, \quad A_{n}(1)=n !
$$

For example,

$$
\begin{gathered}
A_{0}(x)=1, \quad A_{1}(x)=x, \quad A_{2}(x)=x+x^{2}, \quad A_{3}(x)=x+4 x^{2}+x^{3}, \\
A_{4}(x)=x+11 x^{2}+11 x^{3}+x^{4} .
\end{gathered}
$$


In this connection, we will called polynomials $g_{n}(x)$ the generalized Euler polynomials.

"Square" Riordan matrices (called convolution arrays) and numerator polynomials of the generating functions of their rows were considered in the series of papers [7] - [11]. In [12] such matrices are called generalized Riordan arrays. Concept of generalized Euler polynomials (called $p_{n}$-associated Eulerian polynomials) in general form is represented in [13].

Mentioned series of papers was written before the advent of the Riordan matrices theory. In modern literature, the topic of numerator polynomials of the Riordan matrices has not been raised. Exception consists of the three works [14] - [16], in which the generating functions of the diagonals of exponential Riordan matrices are considered. These works provided an incentive for writing the present paper.

In paper, we study the transformations in which the numerator polynomials of Riordan matrices participate, and build matrices of these transformations according to certain rules. These matrices serve as tools for further research. In Section 2, we consider the numerator polynomials of ordinary Riordan matrices (generalized Euler polynomials) and the transformations associated with them. Using matrices of these transformations, we obtain a general formula for the numerator polynomials of the matrices $\left(1, x_{(\beta)} a(x)\right)$, where ${ }_{(\beta)} a(x)$ is the generalized binomial series:

$$
{ }_{(\beta)} a^{\varphi}(x)=\sum_{n=0}^{\infty} \frac{\varphi}{\varphi+\beta n}\left(\begin{array}{c}
\varphi+\beta n \\
n
\end{array}\right) x^{n} .
$$

In Section 3, we consider the numerator polynomials of exponential Riordan matrices (generalized Narayana polynomials) and the transformations associated with them. In Section 4, we introduce matrices of the operators mapping generalized Euler polynomials to generalized Narayana polynomials. Using these matrices, we obtain a general formula for the numerator polynomials of the matrices $\left(1, x_{(\beta)} a(x)\right)_{E}$, where $(\beta) a(x)$ is the generalized binomial series. In Section 5, we give an idea of the generalized Lagrange series ${ }_{(\beta)} a(x)$ defined by the identity ${ }_{(\beta)} a(x)=a\left(x_{(\beta)} a^{\beta}(x)\right)$, or $\left(1, x a^{-\beta}(x)\right)^{-1}=\left(1, x_{(\beta)} a^{\beta}(x)\right)$. In other words, we extend the generalization that underlies the generalized binomial series to any formal power series $a(x), a_{0}=1$. In the remainder of paper, we will consider the transformations associated with these series.

\section{Generalized Euler polynomials}

In [13], the transformations associated with the generalized Euler polynomials are presented from a general point of view. In this section, we consider them from point of view of the Riordan matrix theory. We denote

$$
\begin{gathered}
{[n, \rightarrow](b(x), \log a(x))=c_{n}(x)=\sum_{m=0}^{n} c_{m} x^{m}, \quad b_{0} \neq 0, \quad a_{0}=1,} \\
{[n, \rightarrow](b(x), \log a(x))_{E}=s_{n}(x)=\sum_{m=0}^{n} s_{m} x^{m}, \quad[n, \rightarrow](b(x), a(x))=\frac{g_{n}(x)}{(1-x)^{n+1}} .}
\end{gathered}
$$

Then

$$
b(x) a^{m}(x)=\sum_{n=0}^{\infty} \frac{s_{n}(m)}{n !} x^{n}, \quad \frac{g_{n}(x)}{(1-x)^{n+1}}=\sum_{m=0}^{\infty} \frac{s_{n}(m)}{n !} x^{m}
$$

Since

$$
(b(x), a(x))=(b(x), \log a(x))\left(1, e^{x}\right),
$$


then

$$
\frac{g_{n}(x)}{(1-x)^{n+1}}=\sum_{p=0}^{n} \frac{c_{p} A_{p}(x) / p !}{(1-x)^{p+1}}=\frac{1}{n !} \sum_{p=0}^{n} \frac{s_{p} A_{p}(x)}{(1-x)^{p+1}}=\frac{\frac{1}{n !} \sum_{p=0}^{n} s_{p}(1-x)^{n-p} A_{p}(x)}{(1-x)^{n+1}} .
$$

We introduce the matrices $U_{n}$ :

$$
U_{n} x^{p}=(1-x)^{n+1} \frac{1}{n !} \sum_{m=0}^{\infty} m^{p} x^{m}=\frac{1}{n !}(1-x)^{n-p} A_{p}(x), \quad 0 \leq p \leq n .
$$

For example,

$$
U_{1}=\left(\begin{array}{cc}
1 & 0 \\
-1 & 1
\end{array}\right), \quad U_{2}=\frac{1}{2 !}\left(\begin{array}{ccc}
1 & 0 & 0 \\
-2 & 1 & 1 \\
1 & -1 & 1
\end{array}\right), \quad U_{3}=\frac{1}{3 !}\left(\begin{array}{cccc}
1 & 0 & 0 & 0 \\
-3 & 1 & 1 & 1 \\
3 & -2 & 0 & 4 \\
-1 & 1 & -1 & 1
\end{array}\right)
$$

Then $U_{n} s_{n}(x)=g_{n}(x)$. Let the symbols $(\varphi)_{n},[\varphi]_{n}$ denote respectively the descending and ascending factorial:

$$
(\varphi)_{n}=\varphi(\varphi-1) \ldots(\varphi-n+1), \quad[\varphi]_{n}=\varphi(\varphi+1) \ldots(\varphi+n-1) .
$$

Since

$$
\frac{x^{p}}{(1-x)^{n+1}}=\sum_{m=0}^{\infty}\left(\begin{array}{c}
m+n-p \\
n
\end{array}\right) x^{m}=\sum_{m=0}^{\infty} \frac{(m+n-p)_{n}}{n !} x^{m}, \quad 0 \leq p \leq n,
$$

then

$$
U_{n}^{-1} x^{p}=(x+n-p)_{n}=(x)_{p}[x+1]_{n-p} .
$$

For example,

$$
U_{1}^{-1}=\left(\begin{array}{cc}
1 & 0 \\
1 & 1
\end{array}\right), \quad U_{2}^{-1}=\left(\begin{array}{ccc}
2 & 0 & 0 \\
3 & 1 & -1 \\
1 & 1 & 1
\end{array}\right), \quad U_{3}^{-1}=\left(\begin{array}{cccc}
6 & 0 & 0 & 0 \\
11 & 2 & -1 & 2 \\
6 & 3 & 0 & -3 \\
1 & 1 & 1 & 1
\end{array}\right) .
$$

We will consider the products of matrices of various orders with infinite matrices. Thus, the matrix of order $n$ corresponds to infinite matrix, the columns elements of which, starting from the $n$th column, $n=0,1,2, \ldots$, and the rows elements of which, starting from the $n$th row, are equal to zero. We introduce the matrices $J_{n}$ corresponding to the operator rearranging a coefficients of polynomial of degree $n$ in the reverse order: $J_{n} c(x)=x^{n} c(1 / x)$, where $c(x)$ is a polynomial of degree $\leq n$. For example,

$$
J_{3}=\left(\begin{array}{llll}
0 & 0 & 0 & 1 \\
0 & 0 & 1 & 0 \\
0 & 1 & 0 & 0 \\
1 & 0 & 0 & 0
\end{array}\right)
$$

Theorem 2.1.

$$
U_{n} E(1,-x) U_{n}^{-1}=(-1)^{n} J_{n}
$$

Proof.

$$
E(1,-x)(x)_{p}[x+1]_{n-p}=(-x-1)_{p}[-x]_{n-p}=(-1)^{n}(x)_{n-p}[x+1]_{p},
$$




$$
E(1,-x) U_{n}^{-1}=(-1)^{n} U_{n}^{-1} J_{n}
$$

Theorem 2.2. Polynomials $(-1)^{n} J_{n} g_{n}(x)$ are the numerator polynomials of the matrix $\left(b(x) a^{-1}(x), x a^{-1}(x)\right)$.

Proof. By the Theorem 2.1.

$$
(-1)^{n} J_{n} g_{n}(x)=U_{n} E(1,-x) s_{n}(x) .
$$

Since

$$
(b(x), \log a(x))(1,-x)\left(e^{x}, x\right)=\left(b(x) a^{-1}(x), \log a^{-1}(x)\right)
$$

then

$$
E(1,-x) s(x)=s_{n}(-x-1)=[n, \rightarrow]\left(b(x) a^{-1}(x), \log a^{-1}(x)\right)_{E} .
$$

\section{Theorem 2.3.}

$$
g_{n}(1)=b_{0}\left(a_{1}\right)^{n} .
$$

Proof. Denote $U_{n} x^{p}=U_{n, p}(x)$. Since

$a_{1}=[x] \log a(x), \quad b_{0}\left(a_{1}\right)^{n}=\left[x^{n}\right] s_{n}(x) ; \quad U_{n, p}(1)=0, \quad p<n ; \quad U_{n, n}(1)=1$, then

$$
g_{n}(1)=\sum_{p=0}^{n} s_{p} U_{n, p}(1)=s_{n}=b_{0}\left(a_{1}\right)^{n} .
$$

Case, when $a_{1}=0$ and the degree of polynomial $s_{n}(x)$ is less than $n$, is possible. This possibility is reflected in the following theorem.

Theorem 2.4. If $c_{n-m}(x)$ is a polynomial of degree $n-m$, then

$$
U_{n} c_{n-m}(x)=(1-x)^{m} \frac{(n-m) !}{n !} U_{n-m} c_{n-m}(x) .
$$

Respectively, if $d_{n-m}(x)$ is a polynomial of degree $\leq n-m$, then

$$
U_{n}^{-1}(1-x)^{m} d_{n-m}(x)=\frac{n !}{(n-m) !} U_{n-m}^{-1} d_{n-m}(x) .
$$

Proof. Let $I_{n}$ is the identity matrix of order $n+1$. It is obvious that

$$
\left((1-x)^{-m}, x\right) U_{n} I_{n-m}=\frac{(n-m) !}{n !} U_{n-m},
$$

or

$$
U_{n} I_{n-m}=\left((1-x)^{m}, x\right) \frac{(n-m) !}{n !} U_{n-m}
$$

Respectively,

$$
U_{n}^{-1}\left((1-x)^{m}, x\right) I_{n-m}=\frac{n !}{(n-m) !} U_{n-m}^{-1} .
$$

We introduce the matrices $V_{n}=J_{n} E J_{n}=\left((1+x)^{n+1}, x\right) P^{-1} I_{n}$. For example,

$$
V_{3}=\left(\begin{array}{cccc}
1 & 0 & 0 & 0 \\
3 & 1 & 0 & 0 \\
3 & 2 & 1 & 0 \\
1 & 1 & 1 & 1
\end{array}\right), \quad V_{3}^{-1}=\left(\begin{array}{cccc}
1 & 0 & 0 & 0 \\
-3 & 1 & 0 & 0 \\
3 & -2 & 1 & 0 \\
-1 & 1 & -1 & 1
\end{array}\right)
$$


If $c(x)$ is a polynomial of degree $\leq n$, then

$$
V_{n} c(x)=(1+x)^{n} c\left(\frac{x}{1+x}\right), \quad V_{n}^{-1} c(x)=(1-x)^{n} c\left(\frac{x}{1-x}\right) .
$$

We found in the introduction that $V_{n}^{-1} w_{n}(x)=g_{n}(x)$, where $w_{n}(x)=[n, \rightarrow](b(x), a(x)-1)$, and hence $U_{n}^{-1} V_{n}^{-1} w_{n}(x)=s_{n}(x)$. By the Theorem 2.4. we find:

$$
U_{n}^{-1} V_{n}^{-1} x^{p}=U_{n}^{-1}(1-x)^{n-p} x^{p}=\frac{n !}{p !}(x)_{p}=\frac{n !}{p !} \sum_{m=0}^{p} s(p, m) x^{m},
$$

where $s(p, m)$ are the Stirling numbers of the first kind. Hence

$$
V_{n} U_{n} x^{p}=\frac{1}{n !} \sum_{m=0}^{p} m ! S(p, m) x^{m},
$$

where $S(p, m)$ are the Stirling numbers of the second kind. For example, $U_{3}^{-1} V_{3}^{-1}, V_{3} U_{3}$ :

$$
3 !\left(\begin{array}{cccc}
1 & 0 & 0 & 0 \\
0 & 1 & -1 & 2 \\
0 & 0 & 1 & -3 \\
0 & 0 & 0 & 1
\end{array}\right)\left(\begin{array}{cccc}
1 & 0 & 0 & 0 \\
0 & 1 & 0 & 0 \\
0 & 0 & \frac{1}{2 !} & 0 \\
0 & 0 & 0 & \frac{1}{3 !}
\end{array}\right), \quad \frac{1}{3 !}\left(\begin{array}{cccc}
1 & 0 & 0 & 0 \\
0 & 1 & 0 & 0 \\
0 & 0 & 2 ! & 0 \\
0 & 0 & 0 & 3 !
\end{array}\right)\left(\begin{array}{cccc}
1 & 0 & 0 & 0 \\
0 & 1 & 1 & 1 \\
0 & 0 & 1 & 3 \\
0 & 0 & 0 & 1
\end{array}\right) .
$$

Numerator polynomials of the matrix $(1, x a(x))$ are of independent interest. We introduce special notation for them and the associated polynomials:

$$
\begin{gathered}
{[n, \searrow](1, x a(x))=\frac{\alpha_{n}(x)}{(1-x)^{n+1}}, \quad[n, \searrow]\left(1, x a^{-1}(x)\right)=\frac{\alpha_{n}^{(-1)}(x)}{(1-x)^{n+1}},} \\
{[n, \rightarrow](1, \log a(x))_{E}=u_{n}(x), \quad[n, \rightarrow](1, a(x)-1)=v_{n}(x) .}
\end{gathered}
$$

Since $[n, \searrow]\left(a^{-1}(x), x a^{-1}(x)\right)=(1 / x)[n, \searrow]\left(1, x a^{-1}(x)\right), n>0$, then by the Theorem 2. 2.

$$
\alpha_{n}^{(-1)}(x)=(-1)^{n} x J_{n} \alpha_{n}(x) .
$$

Example 2.1.

$$
\begin{gathered}
a(x)=\frac{1+x}{1-x}, \quad a(x)-1=\frac{2 x}{1-x}, \quad v_{n}(x)=2^{n} x\left(\frac{1}{2}+x\right)^{n-1}, \quad n>0, \\
\alpha_{n}(x)=V_{n}^{-1} v_{n}(x)=2 x(1+x)^{n-1}, \\
u_{n}(x)=U_{n}^{-1} \alpha_{n}(x)=2 \sum_{p=0}^{n}\left(\begin{array}{c}
n-1 \\
p-1
\end{array}\right)(x)_{p}[x+1]_{n-p}= \\
=U_{n}^{-1} V_{n}^{-1} v_{n}(x)=n ! \sum_{p=0}^{n}\left(\begin{array}{c}
n-1 \\
p-1
\end{array}\right) \frac{2^{p}}{p !}(x)_{p} .
\end{gathered}
$$

Example 2.2. Let $(1, x g(x))^{-1}=\left(1, x b^{-1}(x)\right), g_{0}=1, b_{0}=1$. Then by the Lagrange inversion theorem

$$
\left[x^{n}\right] g^{m}(x)=\frac{m}{m+n}\left[x^{n}\right] b^{m+n}(x)=\left[x^{n}\right]\left(1-x(\log b(x))^{\prime}\right) b^{m+n}(x) .
$$

Denote $\left[x^{n}\right] g^{m}(x)=g_{n}^{(m)},\left[x^{n}\right]\left(1-x(\log b(x))^{\prime}\right) b^{m}(x)=c_{n}^{(m)}$. Then

$$
[n, \rightarrow](1, x g(x))=\sum_{m=0}^{n} g_{n-m}^{(m)} x^{m}=\sum_{m=0}^{n} c_{n-m}^{(n)} x^{m}=[n, \rightarrow]\left(\left(1-x(\log b(x))^{\prime}\right) b^{n}(x), x\right)
$$


If

$$
g(x)=\frac{x}{2}+\sqrt{1+\frac{x^{2}}{4}}
$$

then

$$
\begin{aligned}
& b(x)=\sqrt{1+x}, \quad 1-x(\log b(x))^{\prime}=\left(1+\frac{x}{2}\right) \frac{1}{1+x}, \\
& {[2 n, \rightarrow](1, x g(x))=\left(\frac{1}{2}+x\right) x^{n}(1+x)^{n-1}, \quad n>0 .}
\end{aligned}
$$

Let

$$
a(x)=\left(\frac{x}{2}+\sqrt{1+\frac{x^{2}}{4}}\right)^{2} .
$$

Then

$$
\begin{gathered}
a(x)-1=x \sqrt{a(x)}=x\left(\frac{x}{2}+\sqrt{1+\frac{x^{2}}{4}}\right) \\
v_{2 n}(x)=\left(\frac{1}{2}+x\right) x^{n}(1+x)^{n-1}, \quad \alpha_{2 n}(x)=V_{2 n}^{-1} v_{2 n}(x)=\frac{1}{2}(1+x) x^{n}, \\
u_{2 n}(x)=U_{2 n}^{-1} \alpha_{2 n}(x)=\frac{1}{2}(x)_{n}[x+1]_{n}+\frac{1}{2}(x)_{n+1}[x+1]_{n-1}=\prod_{m=0}^{n-1}\left(x^{2}-m^{2}\right) .
\end{gathered}
$$

We find the generating function of the sequence of polynomials $\alpha_{n}(t)$. Since

$$
\frac{\alpha_{n}(t)}{(1-t)^{n+1}}=\sum_{m=0}^{\infty}\left[x^{n}\right] a^{m}(x) t^{m}=\left[x^{n}\right] \frac{1}{1-t a(x)},
$$

then

$$
\sum_{n=0}^{\infty} \alpha_{n}(t) x^{n}=\frac{1-t}{1-t a(x(1-t))} .
$$

Example 2.3. This example is a generalization of the examples considered in [11]. Denote $\tilde{\alpha}_{0}(x)=1, \tilde{\alpha}_{n}(x)=(1 / x) \alpha_{n}(x)$. If

$$
[n, \searrow]\left(1, \frac{x}{1+\varphi x+\beta x^{2}}\right)=\frac{\alpha_{n}(x)}{(1-x)^{n+1}},
$$

then

$$
\tilde{\alpha}_{n}(x)=[n, \rightarrow]\left(\frac{1}{1+\varphi x+\beta x^{2}}, \frac{\beta x^{2}}{1+\varphi x+\beta x^{2}}\right) .
$$

Really,

$$
\begin{gathered}
1+\sum_{n=1}^{\infty} \tilde{\alpha}_{n}(t) x^{n}=\left(\frac{1}{1+\varphi x+\beta x^{2}}, \frac{\beta x^{2}}{1+\varphi x+\beta x^{2}}\right) \frac{1}{1-t x}=1+\frac{-\varphi x-\beta(1-t) x^{2}}{1+\varphi x+\beta(1-t) x^{2}}, \\
\sum_{n=0}^{\infty} \alpha_{n}(t) x^{n}=1+t \sum_{n=1}^{\infty} \tilde{\alpha}_{n}(t) x^{n}=\frac{1+\varphi(1-t) x+\beta(1-t)^{2} x^{2}}{1+\varphi x+\beta(1-t) x^{2}},
\end{gathered}
$$

that corresponds to the formula (1).

Let ${ }_{(\beta)} a(x)$ is the generalized binomial series:

$$
{ }_{(\beta)} a^{\varphi}(x)=\sum_{n=0}^{\infty} \frac{\varphi}{\varphi+n \beta}\left(\begin{array}{c}
\varphi+n \beta \\
n
\end{array}\right) x^{n}
$$




$$
\begin{gathered}
{ }_{(0)} a(x)=1+x, \quad{ }_{(1)} a(x)=\frac{1}{1-x}, \quad{ }_{(2)} a(x)=\frac{1-\sqrt{1-4 x}}{2 x}, \\
{ }_{(-1)} a(x)=\frac{1+\sqrt{1+4 x}}{2}, \quad{ }_{(1 / 2)} a(x)=\left(\frac{x}{2}+\sqrt{1+\frac{x^{2}}{4}}\right)^{2} .
\end{gathered}
$$

Denote

$$
\begin{gathered}
{[n, \searrow]\left(1, x_{(\beta)} a(x)\right)=\frac{{ }_{(\beta)} \alpha_{n}(x)}{(1-x)^{n+1}}=\sum_{m=0}^{\infty} \frac{m}{m+n \beta}\left(\begin{array}{c}
m+n \beta \\
n
\end{array}\right) x^{m},} \\
{[n, \rightarrow]\left(1,,_{(\beta)} a(x)-1\right)={ }_{(\beta)} v_{n}(x) .}
\end{gathered}
$$

\section{Theorem 2.5.}

$$
{ }_{(\beta)} \alpha_{n}(x)=\frac{1}{n} \sum_{m=0}^{n}\left(\begin{array}{c}
n(1-\beta) \\
m-1
\end{array}\right)\left(\begin{array}{c}
n \beta \\
n-m
\end{array}\right) x^{m}, \quad n>0 .
$$

Proof. We take into account the property of generalized binomial series: ${ }_{(\beta)} a(x)-1=$ $x_{(\beta)} a^{\beta}(x)$. Then

$$
{ }_{(\beta)} v_{n}(x)=[n, \rightarrow]\left(1, x_{(\beta)} a^{\beta}(x)\right)=\sum_{m=0}^{n} \frac{m}{n}\left(\begin{array}{c}
n \beta \\
n-m
\end{array}\right) x^{m} .
$$

We use the factorial representation of binomial coefficients, i.e., we prove the theorem for positive integers $\beta$. By polynomial argument (binomial coefficients under consideration are polynomials in $\beta$ ) this is equivalent to the general proof. Since

$$
[m, \rightarrow] V_{n}^{-1}=\sum_{i=0}^{m}\left(\begin{array}{c}
m-n-1 \\
m-i
\end{array}\right) x^{i}=\sum_{i=0}^{m}(-1)^{m-i}\left(\begin{array}{c}
n-i \\
m-i
\end{array}\right) x^{i}
$$

then

$$
\begin{gathered}
{\left[x^{m}\right]_{(\beta)} \alpha_{n}(x)=\left[x^{m}\right] V_{n}^{-1}(\beta)} \\
v_{n}(x)= \\
=\sum_{i=0}^{m}(-1)^{m-i}\left(\begin{array}{c}
n-i \\
m-i
\end{array}\right) \frac{i}{n}\left(\begin{array}{c}
n \beta \\
n-i
\end{array}\right) \frac{(n \beta-n+m) !}{(n \beta-n+m) !}= \\
=\frac{1}{n}\left(\begin{array}{c}
n \beta \\
n-m
\end{array}\right) \sum_{i=0}^{m}(-1)^{m-i} i\left(\begin{array}{c}
n \beta-n+m \\
m-i
\end{array}\right)= \\
=\frac{1}{n}\left(\begin{array}{c}
n \beta \\
n-m
\end{array}\right)(-1)^{m-1}\left(\begin{array}{c}
n \beta-n+m-2 \\
m-1
\end{array}\right)=\frac{1}{n}\left(\begin{array}{c}
n \beta \\
n-m
\end{array}\right)\left(\begin{array}{c}
n(1-\beta) \\
m-1
\end{array}\right) .
\end{gathered}
$$

Note that

$$
{ }_{(0)} \alpha_{n}(x)=x^{n}, \quad{ }_{(1)} \alpha_{n}(x)=x, \quad{ }_{(1 / 2)} \alpha_{2 n}(x)=\frac{1}{2}(1+x) x^{n} .
$$

Since ${ }_{(1-\beta)} a(x)={ }_{(\beta)} a^{-1}(-x)$, then ${ }_{(1-\beta)} \alpha_{n}(x)=x J_{n(\beta)} \alpha_{n}(x)$.

\section{Generalized Narayana polynomials}

Constructive relationships between the ordinary and exponential Riordan matrices exist. Particular manifestations of these relationships resemble the details of construction, the general plan of which is a secret for us. Following [14] - [16], we will consider some of such manifestations associated with the numerator polynomials. Since

$$
[n, \searrow](b(x), x a(x))=\sum_{m=0}^{\infty} \frac{s_{n}(m)}{n !} x^{m},
$$


then

$$
[n, \searrow](b(x), x a(x))_{E}=\sum_{m=0}^{\infty} \frac{(m+n) !}{m !} \frac{s_{n}(m)}{n !} x^{m}=\sum_{m=0}^{\infty} \frac{[m+1]_{n} s_{n}(m)}{n !} x^{m} .
$$

If $a_{1} \neq 0$, then $[x+1]_{n} s_{n}(x)$ is the polynomial of degree $2 n$, in general case - of degree $\leq 2 n$, so that

$$
\sum_{m=0}^{\infty} \frac{[m+1]_{n} s_{n}(m)}{n !} x^{m}=\frac{h_{n}(x)}{(1-x)^{2 n+1}}, \quad h_{n}(x)=\frac{(2 n) !}{n !} U_{2 n}[x+1]_{n} s_{n}(x) .
$$

Since $[-m]_{n}=0$ when $m=0,1, \ldots, n-1$, then in accordance with the Theorem 2.2.

$$
\sum_{m=0}^{\infty} \frac{[-m]_{n} s_{n}(-m-1)}{n !} x^{m}=\frac{(-1)^{2 n} J_{2 n} h_{n}(x)}{(1-x)^{2 n+1}}=\frac{(-1)^{2 n} x^{n} J_{n} h_{n}(x)}{(1-x)^{2 n+1}},
$$

i.e., $h_{n}(x)$ is the polynomial of degree $\leq n$. Since $\left[x^{2 n}\right][x+1]_{n} s_{n}(x)=b_{0}\left(a_{1}\right)^{n}$, then in accordance with the Theorem 2.3.

$$
h_{n}(1)=b_{0}\left(a_{1}\right)^{n} \frac{(2 n) !}{n !} .
$$

If $b(x)=1, a(x)=(1-x)^{-1}$, then

$$
h_{n}(x)=(n+1) ! N_{n}(x)=(1-x)^{2 n+1} \sum_{m=0}^{\infty}[m+1]_{n}\left(\begin{array}{c}
m+n-1 \\
n
\end{array}\right) x^{m},
$$

where

$$
N_{0}(x)=1, \quad N_{n}(x)=\frac{1}{n} \sum_{m=0}^{n}\left(\begin{array}{c}
n \\
m-1
\end{array}\right)\left(\begin{array}{c}
n \\
n-m
\end{array}\right) x^{m}
$$

are the Narayana polynomials. In this connection, we will called polynomials $h_{n}(x)$ the generalized Narayana polynomials.

We introduce the matrices $F_{n}$ :

$$
\begin{gathered}
F_{n}=\frac{(2 n) !}{n !} U_{2 n}\left([x+1]_{n}, x\right) I_{n}, \quad F_{n}^{-1}=\frac{n !}{(2 n) !}\left([x+1]_{n}, x\right)^{-1} U_{2 n}^{-1} I_{n}, \\
F_{n} x^{p}=\frac{(2 n) !}{n !} U_{2 n} x^{p}[x+1]_{n}=(1-x)^{2 n+1} \sum_{m=0}^{\infty} m^{p}\left(\begin{array}{c}
m+n \\
n
\end{array}\right) x^{m}, \\
F_{n}^{-1} x^{p}=\frac{n !}{(2 n) !}(x)_{p}[x+n+1]_{n-p} .
\end{gathered}
$$

For example,

$$
\begin{gathered}
F_{1}=\left(\begin{array}{cc}
1 & 0 \\
-1 & 2
\end{array}\right), \quad F_{2}=\left(\begin{array}{ccc}
1 & 0 & 0 \\
-2 & 3 & 3 \\
1 & -3 & 9
\end{array}\right), \quad F_{3}=\left(\begin{array}{cccc}
1 & 0 & 0 & 0 \\
-3 & 4 & 4 & 4 \\
3 & -8 & 12 & 52 \\
-1 & 4 & -16 & 64
\end{array}\right) ; \\
F_{1}^{-1}=\frac{1}{2 !}\left(\begin{array}{ll}
2 & 0 \\
1 & 1
\end{array}\right), \quad F_{2}^{-1}=\frac{2 !}{4 !}\left(\begin{array}{ccc}
12 & 0 & 0 \\
7 & 3 & -1 \\
1 & 1 & 1
\end{array}\right), \quad F_{3}^{-1}=\frac{3 !}{6 !}\left(\begin{array}{cccc}
120 & 0 & 0 & 0 \\
74 & 20 & -4 & 2 \\
15 & 9 & 3 & -3 \\
1 & 1 & 1 & 1
\end{array}\right) .
\end{gathered}
$$


Then $F_{n} s_{n}(x)=h_{n}(x)$.

Example 3.1. We explain the identity

$$
F_{n}[x+n+1]_{n}=\frac{(2 n) !}{n !} .
$$

Matrix $\left((x a(x))^{\prime}, x a(x)\right)_{E}$ is similar to the matrix $(a(x), x a(x))$ in the sense that $[n, \searrow]\left((x a(x))^{\prime}, x a(x)\right)_{E}=(1 / x)[n, \searrow](1, x a(x))_{E}, n>0$. This is a consequence of the identities

$$
\begin{gathered}
{\left[x^{n}\right]\left(1+x(\log a(x))^{\prime}\right) a^{m}(x)=\frac{m+n}{m}\left[x^{n}\right] a^{m}(x),} \\
{\left[x^{n}\right](x a(x))^{\prime} a^{m}(x)=\frac{m+n+1}{m+1}\left[x^{n}\right] a^{m+1}(x) .}
\end{gathered}
$$

It also shows that

$$
[n, \rightarrow]\left((x a(x))^{\prime}, \log a(x)\right)_{E}=(x+n+1) \tilde{u}_{n}(x+1), \quad n>0,
$$

where $\tilde{u}_{n}(x)=(1 / x) u_{n}(x)$. If $a(x)=C(x)$, where $C(x)$ is the Catalan series, then

$$
\tilde{u}_{n}(x)=[x+n+1]_{n-1}, \quad(x+n+1) \tilde{u}_{n}(x+1)=[x+n+1]_{n} .
$$

Hence, numerator polynomials of the matrix $(1, x C(x))_{E}$ are the monomials $((2 n) ! / n !) x$.

Theorem 3.1.

$$
F_{n} E^{n+1}(1,-x) F_{n}^{-1}=(-1)^{n} J_{n}
$$

\section{Proof.}

$$
E^{n+1}(1,-x)(x)_{p}[x+n+1]_{n-p}=(-x-n-1)_{p}[-x]_{n-p}=(-1)^{n}(x)_{n-p}[x+n+1]_{p},
$$

or

$$
E^{n+1}(1,-x) F_{n}^{-1}=(-1)^{n} F_{n}^{-1} J_{n} .
$$

Denote $(1, x a(x))^{-1}=(1, x \bar{a}(x))$.

Theorem 3.2. Polynomials $(-1)^{n} J_{n} h_{n}(x)$ are the numerator polynomials of the matrix $\left(b(x \bar{a}(x))(x \bar{a}(x))^{\prime}, x \bar{a}(x)\right)_{E}$.

Proof. By the Theorem 3.1.

$$
(-1)^{n} J_{n} h_{n}(x)=F_{n} s_{n}(-x-n-1) .
$$

We will imagine an infinite table whose $k$ th row, $k=0, \pm 1, \pm 2, \ldots$, has the generating function

$$
b(x) a^{k}(x)=\sum_{n=0}^{\infty} \frac{s_{n}(k)}{n !} x^{n} .
$$

If the row numbers of the table decrease from top to bottom, then, according to the Lagrange inversion theorem, the $k$ th descending diagonal of table has the generating function

$$
b(x \bar{a}(x))\left(1+x(\log \bar{a}(x))^{\prime}\right)\left(\frac{1}{\bar{a}(x)}\right)^{k}=\sum_{n=0}^{\infty} \frac{s_{n}(k-n)}{n !} x^{n} .
$$

Hence,

$$
b(x \bar{a}(x))(x \bar{a}(x))^{\prime}(\bar{a}(x))^{k}=\sum_{n=0}^{\infty} \frac{s_{n}(-k-n-1)}{n !} x^{n} .
$$

Denote

$$
[n, \searrow](1, x a(x))_{E}=\frac{\varphi_{n}(x)}{(1-x)^{2 n+1}}, \quad[n, \searrow](1, x a(x))_{E}^{-1}=\frac{\varphi_{n}^{[-1]}(x)}{(1-x)^{2 n+1}}
$$


Then

$$
\varphi_{n}^{[-1]}(x)=(-1)^{n} x J_{n} \varphi_{n}(x), \quad n>0 .
$$

Hence, if the matrix $(1, x a(x))$ is the pseudo-involution [3], i.e., $(1, x a(x))^{-1}=(1, x a(-x))$, then $\varphi_{n}(x)=x J_{n} \varphi_{n}(x)$.

We will find a specific generating function for the sequence of polynomials $\varphi_{n}(t)$. Since

$$
\begin{gathered}
\frac{\varphi_{n}(t)}{(n+1) !(1-t)^{2 n+1}}=\sum_{m=0}^{\infty} \frac{1}{n+1}\left(\begin{array}{c}
n+m \\
m
\end{array}\right)\left[x^{n}\right] a^{m}(x) t^{m}= \\
=\frac{1}{n+1}\left[x^{n}\right] \frac{1}{(1-t a(x))^{n+1}}=\left[x^{n}\right] b(x),
\end{gathered}
$$

where according to the Lagrange inversion theorem

$$
b(x)=\frac{1}{1-t a(x b(x))}, \quad(1, x b(x))=(1, x(1-t a(x)))^{-1},
$$

then

$$
\sum_{n=0}^{\infty} \varphi_{n}(t) \frac{x^{n}}{(n+1) !}=(1-t) b\left(x(1-t)^{2}\right) .
$$

Example 3.2.

$$
\begin{gathered}
a(x)=\frac{1}{1-x}, \quad b(x)=\frac{1+x-t-\sqrt{1-2 x-2 t-2 x t+x^{2}+t^{2}}}{2 x}, \\
\sum_{n=0}^{\infty} \varphi_{n}(t) \frac{x^{n}}{(n+1) !}=\sum_{n=0}^{\infty} N_{n}(t) x^{n}=\frac{1+x(1-t)-\sqrt{1-2 x(1+t)+x^{2}(1-t)^{2}}}{2 x} .
\end{gathered}
$$

Generating functions of the second-order Euler numbers which are the coefficients of numerator polynomials of the matrices $\left(1, e^{x}-1\right)_{E},\left(e^{x}, e^{x}-1\right)_{E}$ are considered in [17].

\section{Connection matrices}

We introduce the matrices $S_{n}=F_{n} U_{n}^{-1}, S_{n}^{-1}=U_{n} F_{n}^{-1}$. For example,

$$
\begin{aligned}
& S_{1}=\left(\begin{array}{ll}
1 & 0 \\
1 & 2
\end{array}\right), \quad S_{2}=2 !\left(\begin{array}{ccc}
1 & 0 & 0 \\
4 & 3 & 0 \\
1 & 3 & 6
\end{array}\right), \quad S_{3}=3 !\left(\begin{array}{cccc}
1 & 0 & 0 & 0 \\
9 & 4 & 0 & 0 \\
9 & 12 & 10 & 0 \\
1 & 4 & 10 & 20
\end{array}\right), \\
& S_{4}=4 !\left(\begin{array}{ccccc}
1 & 0 & 0 & 0 & 0 \\
16 & 5 & 0 & 0 & 0 \\
36 & 30 & 15 & 0 & 0 \\
16 & 30 & 40 & 35 & 0 \\
1 & 5 & 15 & 35 & 70
\end{array}\right) \\
& S_{1}^{-1}=\frac{1}{2 !}\left(\begin{array}{cc}
2 & 0 \\
-1 & 1
\end{array}\right), \quad S_{2}^{-1}=\frac{2 !}{4 !}\left(\begin{array}{ccc}
6 & 0 & 0 \\
-8 & 2 & 0 \\
3 & -1 & 1
\end{array}\right), \quad S_{3}^{-1}=\frac{3 !}{6 !}\left(\begin{array}{cccc}
20 & 0 & 0 & 0 \\
-45 & 5 & 0 & 0 \\
36 & -6 & 2 & 0 \\
-10 & 2 & -1 & 1
\end{array}\right) \text {, } \\
& S_{4}^{-1}=\frac{4 !}{8 !}\left(\begin{array}{ccccc}
70 & 0 & 0 & 0 & 0 \\
-224 & 14 & 0 & 0 & 0 \\
280 & -28 & 14 / 3 & 0 & 0 \\
-160 & 20 & -16 / 3 & 2 & 0 \\
35 & -5 & 5 / 3 & -1 & 1
\end{array}\right)
\end{aligned}
$$


Then $S_{n} g_{n}(x)=h_{n}(x)$.

Theorem 4.1.

$$
S_{n}=V_{n}^{-1} C_{n} V_{n}, \quad C_{n} x^{p}=\frac{(n+p) !}{p !} x^{p} .
$$

Proof. We use the Theorem 2.4. and the identities

$$
U_{n}^{-1} V_{n}^{-1} x^{p}=\frac{n !}{p !}(x)_{p}, \quad U_{n+p}^{-1} x^{p}=(x)_{p}[x+1]_{n} .
$$

Then

$$
\begin{gathered}
F_{n} U_{n}^{-1} V_{n}^{-1} x^{p}=\frac{(2 n) !}{n !} U_{2 n} \frac{n !}{p !}(x)_{p}[x+1]_{n}= \\
=\frac{(2 n) !}{p !}(1-x)^{n-p} \frac{(n+p) !}{(2 n) !} U_{n+p}(x)_{p}[x+1]_{n}=\frac{(n+p) !}{p !}(1-x)^{n-p} x^{p}
\end{gathered}
$$

or

$$
S_{n} V_{n}^{-1}=V_{n}^{-1} C_{n}
$$

For example,

$$
S_{3}=3 !\left(\begin{array}{cccc}
1 & 0 & 0 & 0 \\
-3 & 1 & 0 & 0 \\
3 & -2 & 1 & 0 \\
-1 & 1 & -1 & 1
\end{array}\right)\left(\begin{array}{cccc}
1 & 0 & 0 & 0 \\
0 & 4 & 0 & 0 \\
0 & 0 & 10 & 0 \\
0 & 0 & 0 & 20
\end{array}\right)\left(\begin{array}{cccc}
1 & 0 & 0 & 0 \\
3 & 1 & 0 & 0 \\
3 & 2 & 1 & 0 \\
1 & 1 & 1 & 1
\end{array}\right)
$$

Theorem 4.2.

$$
S_{n} x^{p}=\frac{(n+p) !(n-p) !}{n !} \sum_{m=p}^{n}\left(\begin{array}{c}
n \\
m-p
\end{array}\right)\left(\begin{array}{c}
n \\
n-m
\end{array}\right) x^{m}
$$

Proof.

$$
\begin{gathered}
{[m, \rightarrow] V_{n}^{-1}=\sum_{i=0}^{m}\left(\begin{array}{c}
m-n-1 \\
m-i
\end{array}\right) x^{i}=\sum_{i=0}^{m}(-1)^{m-i}\left(\begin{array}{c}
n-i \\
m-i
\end{array}\right) x^{i},} \\
C_{n} V_{n} x^{p}=\sum_{i=p}^{n} \frac{(n+i) !}{i !}\left(\begin{array}{c}
n-p \\
i-p
\end{array}\right) x^{i} \\
{\left[x^{m}\right] V_{n}^{-1} C_{n} V_{n} x^{p}=\sum_{i=p}^{m}(-1)^{m-i}\left(\begin{array}{c}
n-i \\
m-i
\end{array}\right) \frac{(n+i) !}{i !}\left(\begin{array}{c}
n-p \\
i-p
\end{array}\right)=} \\
=\frac{(n+p) !(n-p) !}{(n-m) ! m !} \sum_{i=p}^{m}(-1)^{m-i}\left(\begin{array}{c}
n+i \\
i-p
\end{array}\right)\left(\begin{array}{c}
m \\
i
\end{array}\right)= \\
=\frac{(n+p) !(n-p) !}{(n-m) ! m !}(-1)^{m-p}\left(\begin{array}{c}
m-n-p-1 \\
m-p
\end{array}\right)=\frac{(n+p) !(n-p) !}{n !}\left(\begin{array}{c}
n \\
m-p
\end{array}\right)\left(\begin{array}{c}
n \\
n-m
\end{array}\right) .
\end{gathered}
$$

Theorem 4.3.

$$
S_{n}^{-1} x^{p}=\frac{p !(n-p) !}{(2 n) !} \sum_{m=p}^{n}\left(\begin{array}{c}
-n \\
m-p
\end{array}\right)\left(\begin{array}{c}
2 n \\
n-m
\end{array}\right) x^{m} .
$$

Proof.

$$
\begin{gathered}
{\left[x^{m}\right] V_{n}^{-1} C_{n}^{-1} V_{n} x^{p}=\sum_{i=p}^{m}(-1)^{m-i}\left(\begin{array}{c}
n-i \\
m-i
\end{array}\right) \frac{i !}{(n+i) !}\left(\begin{array}{c}
n-p \\
i-p
\end{array}\right)=} \\
=\frac{p !(n-p) !}{(n-m) !(n+m) !} \sum_{i=p}^{m}(-1)^{m-i}\left(\begin{array}{c}
i \\
i-p
\end{array}\right)\left(\begin{array}{c}
n+m \\
m-i
\end{array}\right)=
\end{gathered}
$$




$$
=\frac{p !(n-p) !}{(n-m) !(n+m) !}(-1)^{m-p}\left(\begin{array}{c}
n+m-p-1 \\
m-p
\end{array}\right)=\frac{p !(n-p) !}{(2 n) !}\left(\begin{array}{c}
-n \\
m-p
\end{array}\right)\left(\begin{array}{c}
2 n \\
n-m
\end{array}\right) .
$$

Example 4.1. Numerator polynomials of the Pascal matrix are equal to one. Hence, numerator polynomials of the matrix $\left|e^{x}\right|^{-1} P\left|e^{x}\right|$ are the polynomials $S_{n} x^{0}=n !^{B} N_{n}(x)$, where ${ }^{B} N_{n}(x)$ are the so-called Narayana polynomials of type $B$ :

$$
{ }^{B} N_{n}(x)=\sum_{m=0}^{n}\left(\begin{array}{c}
n \\
m
\end{array}\right)^{2} x^{m}=(1-x)^{2 n+1} \sum_{m=0}^{\infty}\left(\begin{array}{c}
m+n \\
n
\end{array}\right)^{2} x^{m}
$$

Pascal matrix belongs to a subgroup of the Riordan group formed by the matrices of the form $(a(x), x a(x))$ and called the Bell subgroup. Numerator polynomials with the property $h_{n}(x)=J_{n} h_{n}(x)$ will be called symmetric.

Theorem 4.4. In the exponential Bell subgroup, only the matrices $\left|e^{x}\right|^{-1} P^{\varphi}\left|e^{x}\right|$ have the symmetric numerator polynomials.

Proof. Let $h_{n}(x)$ are the numerator polynomials of the matrix $(a(x), x a(x))_{E}$. Since $a(x \bar{a}(x))=1 / \bar{a}(x)$, then by the Theorem 3.2. the polynomials $(-1)^{n} J_{n} h_{n}(x)$ are the numerator polynomials of the matrix

$$
\left(1+x(\log \bar{a}(x))^{\prime}, x \bar{a}(x)\right)_{E}=\left(1+x(\log a(x))^{\prime}, x a(x)\right)_{E}^{-1} .
$$

Thus, the necessary condition for the symmetry of numerator polynomials is the condition $a(x)=1+x(\log a(x))^{\prime}$. It comes down to the condition $a^{2}(x)=(x a(x))^{\prime}$, or $\sum_{m=0}^{n} a_{n-m} a_{m}=(n+1) a_{n}$, that is feasible only in the case $a_{n}=\left(a_{1}\right)^{n}$.

$n$th numerator polynomial of the matrix $A$ (i.e., ordinary or exponential Riordan matrix) will be denoted by $\left[P_{n}(x)\right] A$.

Example 4.2. Since $\left[P_{n}(x)\right](1+x, x(1+x))=x^{n-1}, n>0$, then

$$
\begin{gathered}
{\left[P_{n}(x)\right](1+x, x(1+x))_{E}=S_{n} x^{n-1}=\frac{(2 n) !}{n ! 2}(1+x) x^{n-1}:} \\
(1+x, x(1+x))_{E}=\left|e^{x}\right|^{-1}\left(\begin{array}{ccccccc}
1 & 0 & 0 & 0 & 0 & 0 & \ldots \\
1 & 1 & 0 & 0 & 0 & 0 & \ldots \\
0 & 2 & 1 & 0 & 0 & 0 & \ldots \\
0 & 1 & 3 & 1 & 0 & 0 & \ldots \\
0 & 0 & 3 & 4 & 1 & 0 & \ldots \\
0 & 0 & 1 & 6 & 5 & 1 & \ldots \\
\vdots & \vdots & \vdots & \vdots & \vdots & \vdots & \ddots
\end{array}\right)\left|e^{x}\right| .
\end{gathered}
$$

Since

$$
\left(1+x(\log (1+x))^{\prime}, x(1+x)\right)_{E}^{-1}=\left(1+x(\log C(-x))^{\prime}, x C(-x)\right)_{E}
$$

then

$$
\begin{gathered}
{\left[P_{n}(x)\right]\left(1+x(\log C(x))^{\prime}, x C(x)\right)_{E}=\frac{(2 n) !}{n ! 2}(1+x):} \\
\left(1+x(\log C(x))^{\prime}, x C(x)\right)_{E}=\left|e^{x}\right|^{-1}\left(\begin{array}{ccccccc}
1 & 0 & 0 & 0 & 0 & 0 & \ldots \\
1 & 1 & 0 & 0 & 0 & 0 & \ldots \\
3 & 2 & 1 & 0 & 0 & 0 & \ldots \\
10 & 6 & 3 & 1 & 0 & 0 & \ldots \\
35 & 20 & 10 & 4 & 1 & 0 & \ldots \\
126 & 70 & 35 & 15 & 5 & 1 & \ldots \\
\vdots & \vdots & \vdots & \vdots & \vdots & \vdots & \ddots
\end{array}\right)\left|e^{x}\right| .
\end{gathered}
$$

Example 4.3. Since $\left[P_{n}(x)\right]\left((x C(x))^{\prime}, x C(x)\right)_{E}=(2 n) ! / n !$, then

$$
\left[P_{n}(x)\right]\left((x C(x))^{\prime}, x C(x)\right)=\frac{(2 n) !}{n !} S_{n}^{-1} x^{0}=\sum_{m=0}^{n}\left(\begin{array}{c}
-n \\
m
\end{array}\right)\left(\begin{array}{c}
2 n \\
n-m
\end{array}\right) x^{m}:
$$




$$
\left((x C(x))^{\prime}, x C(x)\right)=\left(\begin{array}{ccccccc}
1 & 0 & 0 & 0 & 0 & 0 & \cdots \\
2 & 1 & 0 & 0 & 0 & 0 & \cdots \\
6 & 3 & 1 & 0 & 0 & 0 & \cdots \\
20 & 10 & 4 & 1 & 0 & 0 & \cdots \\
70 & 35 & 15 & 5 & 1 & 0 & \cdots \\
252 & 126 & 56 & 21 & 6 & 1 & \cdots \\
\vdots & \vdots & \vdots & \vdots & \vdots & \vdots & \ddots
\end{array}\right)
$$

Respectively,

$$
\begin{aligned}
(-1)^{n} \sum_{m=0}^{n}\left(\begin{array}{c}
2 n \\
m
\end{array}\right)\left(\begin{array}{c}
-n \\
n-m
\end{array}\right) x^{m} & =\left[P_{n}(x)\right]\left(1+x(\log \mathrm{C}(x))^{\prime}, x C^{-1}(x)\right): \\
\left(1+x(\log C(x))^{\prime}, x C^{-1}(x)\right) & =\left(\begin{array}{ccccccc}
1 & 0 & 0 & 0 & 0 & 0 & \ldots \\
1 & 1 & 0 & 0 & 0 & 0 & \ldots \\
3 & 0 & 1 & 0 & 0 & 0 & \ldots \\
10 & 1 & -1 & 1 & 0 & 0 & \ldots \\
35 & 4 & 0 & -2 & 1 & 0 & \ldots \\
126 & 15 & 1 & 0 & -3 & 1 & \ldots \\
\vdots & \vdots & \vdots & \vdots & \vdots & \vdots & \ddots
\end{array}\right) .
\end{aligned}
$$

Let ${ }_{(\beta)} a(x)$ is the generalized binomial series. Denote

$$
[n, \searrow]\left(1, x_{(\beta)} a(x)\right)_{E}=\frac{(\beta) \varphi_{n}(x)}{(1-x)^{2 n+1}}=\sum_{m=0}^{\infty} \frac{m}{m+\beta n}\left(\begin{array}{c}
m+\beta n \\
n
\end{array}\right)[m+1]_{n} x^{m} .
$$

\section{Theorem 4.5.}

$$
{ }_{(\beta)} \varphi_{n}(x)=\frac{(n+1) !}{n} \sum_{m=0}^{n}\left(\begin{array}{c}
n(2-\beta) \\
m-1
\end{array}\right)\left(\begin{array}{c}
n \beta \\
n-m
\end{array}\right) x^{m}, \quad n>0 .
$$

Proof. We prove the theorem by analogy with the proof of Theorem 2.5.

$$
\begin{gathered}
{\left[x^{m}\right]_{(\beta)} \varphi_{n}(x)=\left[x^{m}\right] S_{n(\beta)} \alpha_{n}(x)=\left[x^{m}\right] V_{n}^{-1} C_{n(\beta)} v_{n}(x)=} \\
=\sum_{i=0}^{m}(-1)^{m-i}\left(\begin{array}{c}
n-i \\
m-i
\end{array}\right) \frac{(n+i) !}{i !} \frac{i}{n}\left(\begin{array}{c}
n \beta \\
n-i
\end{array}\right) \frac{(n \beta-n+m) !}{(n \beta-n+m) !}= \\
=\frac{(n+1) !}{n}\left(\begin{array}{c}
n \beta \\
n-m
\end{array}\right) \sum_{i=0}^{m}(-1)^{m-i}\left(\begin{array}{c}
n+i \\
i-1
\end{array}\right)\left(\begin{array}{c}
n \beta-n+m \\
m-i
\end{array}\right)= \\
=\frac{(n+1) !}{n}\left(\begin{array}{c}
n \beta \\
n-m
\end{array}\right)(-1)^{m-1}\left(\begin{array}{c}
n \beta-2 n+m-2 \\
m-1
\end{array}\right)=\frac{(n+1) !}{n}\left(\begin{array}{c}
n \beta \\
n-m
\end{array}\right)\left(\begin{array}{c}
n(2-\beta) \\
m-1
\end{array}\right) .
\end{gathered}
$$

Note that

$$
\text { (0) } \varphi_{n}(x)=\frac{(2 n) !}{n !} x^{n}, \quad \text { (1) } \varphi_{n}(x)=(n+1) ! N_{n}(x), \quad \text { (2) } \varphi_{n}(x)=\frac{(2 n) !}{n !} x .
$$

Since $\left(1, x_{(\beta)} a(x)\right)^{-1}=\left(1, x_{(\beta-1)} a^{-1}(x)\right),{ }_{(\beta-1)} a^{-1}(-x)={ }_{(2-\beta)} a(x)$, then ${ }_{(2-\beta)} \varphi_{n}(x)=$ $x J_{n(\beta)} \varphi_{n}(x)$. 


\section{$5 \quad$ Generalized Lagrange series}

Let ${ }_{(\beta)} a(x)$ is the generalized binomial series. We agree to denote ${ }_{(0)} a(x)=a(x)$. Then $\left(1, x a^{-\beta}(x)\right)^{-1}=\left(1, x_{(\beta)} a^{\beta}(x)\right),\left(1, x_{(\beta)} a^{\varphi}(x)\right)^{-1}=\left(1, x_{(\beta-\varphi)} a^{-\varphi}(x)\right)$. We extend the generalization that underlies this construction to any formal power series $a(x), a_{0}=1$. Let $\left(1, x a^{-1}(x)\right)^{-1}=(1, x b(x))$. Denote $\left[x^{n}\right] a^{m}(x)=a_{n}^{(m)}$. For Riordan matrices, two forms of the Lagrange inversion formula

$$
\begin{aligned}
& f(x)=f_{0}+\sum_{n=1}^{\infty} \frac{x^{n}}{a^{n}(x)} \frac{1}{n}\left[x^{n-1}\right] f^{\prime}(x) a^{n}(x), \\
& \frac{f(x)}{1-x(\log a(x))^{\prime}}=\sum_{n=0}^{\infty} \frac{x^{n}}{a^{n}(x)}\left[x^{n}\right] f(x) a^{n}(x),
\end{aligned}
$$

where $f(x)$ is an arbitrary series, take the form

$$
\begin{gathered}
{[n, \rightarrow]\left(1, x a^{-1}(x)\right)^{-1}=\sum_{m=0}^{n} \frac{m}{n} a_{n-m}^{(n)} x^{m}, \quad n>0} \\
{[n, \rightarrow]\left(1-x(\log a(x))^{\prime}, x a^{-1}(x)\right)^{-1}=\sum_{m=0}^{n} a_{n-m}^{(n)} x^{m}}
\end{gathered}
$$

Hence,

$$
\begin{gathered}
{\left[x^{n}\right] b^{m}(x)=\frac{m}{m+n}\left[x^{n}\right] a^{m+n}(x)=\left[x^{n}\right]\left(1-x(\log a(x))^{\prime}\right) a^{m+n}(x),} \\
{\left[x^{n}\right]\left(1+x(\log b(x))^{\prime}\right) b^{m}(x)=\frac{m+n}{m}\left[x^{n}\right] b^{m}(x)=\left[x^{n}\right] a^{m+n}(x),} \\
\left(1-x(\log a(x))^{\prime}, x a^{-1}(x)\right)^{-1}=\left(1+x(\log b(x))^{\prime}, x b(x)\right) .
\end{gathered}
$$

Denote $\left(1, x a^{-\beta}(x)\right)^{-1}=\left(1, x(\beta) a^{\beta}(x)\right)$. Then

$$
\begin{gathered}
{\left[x^{n}\right]_{(\beta)} a^{\beta m}(x)=\frac{\beta m}{\beta m+\beta n}\left[x^{n}\right] a^{\beta m+\beta n}(x),} \\
{ }_{(\beta)} a^{\varphi}(x)=\sum_{n=0}^{\infty} \frac{\varphi}{\varphi+\beta n} \frac{u_{n}(\varphi+\beta n)}{n !} x^{n}, \quad u_{n}(x)=[n, \rightarrow](1, \log a(x))_{E} .
\end{gathered}
$$

Let $(1, \log a(x))^{-1}=(1, q(x))$. Then

$$
\begin{gathered}
\left(1, \log _{(\beta)} a(x)\right)=\left(1, x_{(\beta)} a^{\beta}(x)\right)(1, \log a(x)) \\
\left(1, \log _{(\beta)} a(x)\right)^{-1}=(1, q(x))\left(1, x a^{-\beta}(x)\right)=\left(1, q(x) e^{-\beta x}\right) .
\end{gathered}
$$

Denote.

$$
{ }_{(\beta)} u_{n}(x)=[n, \rightarrow]\left(1, \log _{(\beta)} a(x)\right)_{E}, \quad{ }_{(\beta)} q_{n}(x)=\left(1, \log _{(\beta)} a(x)\right)_{E}^{-1} x^{n} .
$$

Since $\left(1, q(x) e^{-\beta x}\right)_{E} x^{n}=\left(e^{-\beta n x}, q(x)\right)_{E} x^{n}$, then the system of identities take place:

$$
\begin{gathered}
\text { (३) }_{n}(x)=\frac{x}{x+n \beta} u_{n}(x+n \beta), \quad{ }_{(\beta)} q_{n}(x)=\frac{1}{1+n \beta x} q_{n}\left(\frac{x}{1+n \beta x}\right), \\
\sum_{n=0}^{\infty}(\beta) u_{n}(\varphi){ }_{(\beta)} q_{n}(x)=\frac{1}{1-\varphi x} .
\end{gathered}
$$


Series $_{(\beta)} a(x)$ for integers $\beta=k$ (denoted by $S_{k}(x)$ ) were introduced in [18]. In [19], these series, called generalized Lagrange series, are considered in connection with the Riordan matrices. They have the following visual interpretation. We will imagine the table $\left\{b(x), a^{\varphi}(x)\right\}_{0}$ whose $k$ th row $k=0, \pm 1, \pm 2, \ldots$, has the generating function $b(x) a^{\varphi k}(x)$. Lagrange inversion formula connects the rows of the table with its ascending and descending diagonals in a certain way. For definiteness, we assume that $\varphi>0$ and the table row numbers decrease from top to bottom. We replace the $k$ th row of the table $\left\{b(x), a^{\varphi}(x)\right\}_{0}$ with the $k$ th ascending diagonal, the resulting table will be denoted by $\left\{b(x), a^{\varphi}(x)\right\}_{1}$. We perform the same operation with the table $\left\{b(x), a^{\varphi}(x)\right\}_{1}$, the result will be denoted by $\left\{b(x), a^{\varphi}(x)\right\}_{2}$, etc. Tables obtained in a similar way for descending diagonals are numbered with negative numbers. Then it turns out that the $k$ th row of the table $\left\{b(x), a^{\varphi}(x)\right\}_{v}$ has the generating function

$$
b\left(x_{(v \varphi)} a^{v \varphi}(x)\right)\left(1+x\left(\log _{(v \varphi)} a^{v \varphi}(x)\right)^{\prime}\right){ }_{(v \varphi)} a^{\varphi k}(x),
$$

that can be written as the pair of mutually inverse identities:

$$
\begin{gathered}
\left\{b(x), a^{\varphi}(x)\right\}_{v}=\left\{b\left(x_{(v \varphi)} a^{v \varphi}(x)\right)\left(1+x\left(\log _{(v \varphi)} a^{v \varphi}(x)\right)^{\prime}\right),{ }_{(v \varphi)} a^{\varphi}(x)\right\}_{0} \\
\left\{b\left(x_{(v \varphi)} a^{v \varphi}(x)\right)\left(1+x\left(\log _{(v \varphi)} a^{v \varphi}(x)\right)^{\prime}\right),{ }_{(v \varphi)} a^{\varphi}(x)\right\}_{-v}=\left\{b(x), a^{\varphi}(x)\right\}_{0} .
\end{gathered}
$$

Operators mapping rows of one table to rows of another, and vice versa, correspond to the matrices

$$
\left(1+x\left(\log _{(\beta)} a^{\beta}(x)\right)^{\prime}, x_{(\beta)} a^{\beta}(x)\right), \quad\left(1+x\left(\log a^{-\beta}(x)\right)^{\prime}, x a^{-\beta}(x)\right), \quad \beta=v \varphi .
$$

In the Riordan matrices theory, generalized Lagrange series do not have a generally accepted notation and are present implicitly in the form of various constructions. These constructions are presented in the papers [20] - [26].

\section{Polynomials ${ }_{(\beta)} g_{n}(x)$.}

Let $[n, \rightarrow](b(x), \log a(x))_{E}=s_{n}(x)$. Then by definition of the series ${ }_{(\beta)} a(x)$

$$
[n, \rightarrow]\left(b\left(x_{(\beta)} a^{\beta}(x)\right)\left(1+x\left(\log _{(\beta)} a^{\beta}(x)\right)^{\prime}\right), \log _{(\beta)} a(x)\right)_{E}=s_{n}(x+\beta n) .
$$

Denote

$$
\left[P_{n}(x)\right]\left(b\left(x_{(\beta)} a^{\beta}(x)\right)\left(1+x\left(\log _{(\beta)} a^{\beta}(x)\right)^{\prime}\right), x_{(\beta)} a(x)\right)={ }_{(\beta)} g_{n}(x) .
$$

We introduce the matrices $G_{n}^{\beta}=U_{n} E^{n \beta} U_{n}^{-1}$. Then $G_{n}^{\beta} g_{n}(x)={ }_{(\beta)} g_{n}(x)$. For example,

$$
G_{1}=\left(\begin{array}{cc}
2 & 1 \\
-1 & 0
\end{array}\right), \quad G_{2}=\left(\begin{array}{ccc}
6 & 3 & 1 \\
-8 & -3 & 0 \\
3 & 1 & 0
\end{array}\right), \quad G_{3}=\left(\begin{array}{cccc}
20 & 10 & 4 & 1 \\
-45 & -20 & -6 & 0 \\
36 & 15 & 4 & 0 \\
-10 & -4 & -1 & 0
\end{array}\right)
$$

Since $[x]_{(\beta)} a(x)=a_{1}$, then ${ }_{(\beta)} g_{n}(1)=g_{n}(1)$; therefore, the sum of elements of each column of the matrix $G_{n}^{\beta}$ is equal to one.

Theorem 6.1.

$$
G_{n}^{-\beta}=J_{n} G_{n}^{\beta} J_{n}
$$


Proof. Since $E^{\varphi}(1,-x)=(1,-x) E^{-\varphi}$, then by the Theorem 2.1.

$$
J_{n} U_{n} E^{n \beta} U_{n}^{-1} J_{n}=U_{n} E(1,-x) E^{n \beta} E(1,-x) U_{n}^{-1}=U_{n} E^{-n \beta} U_{n}^{-1} .
$$

Thus,

$$
G_{1}^{-1}=\left(\begin{array}{cc}
0 & -1 \\
1 & 2
\end{array}\right), \quad G_{2}^{-1}=\left(\begin{array}{ccc}
0 & 1 & 3 \\
0 & -3 & -8 \\
1 & 3 & 6
\end{array}\right), \quad G_{3}^{-1}=\left(\begin{array}{cccc}
0 & -1 & -4 & -10 \\
0 & 4 & 15 & 36 \\
0 & -6 & -20 & -45 \\
1 & 4 & 10 & 20
\end{array}\right)
$$

\section{Theorem 6.2.}

$$
G_{n}^{\beta}=V_{n}^{-1}\left((1+x)^{n \beta}, x\right)^{T} V_{n}
$$

Proof. Since

$$
\begin{gathered}
n !\left|e^{x}\right| V_{n} U_{n} x^{p}=[p, \rightarrow]\left(1, e^{x}-1\right)_{E}, \\
(1 / n !) U_{n}^{-1} V_{n}^{-1}\left|e^{x}\right|^{-1} x^{p}=[p, \rightarrow](1, \log (1+x))_{E}, \\
(1, \log (1+x))_{E}\left(e^{n \beta}, x\right)_{E}\left(1, e^{x}-1\right)_{E}=\left((1+x)^{n \beta}, x\right)_{E}
\end{gathered}
$$

then

$$
V_{n} U_{n} E^{n \beta} U_{n}^{-1} V_{n}^{-1}=\left((1+x)^{n \beta}, x\right)^{T} I_{n}
$$

For example,

$$
G_{3}=\left(\begin{array}{cccc}
1 & 0 & 0 & 0 \\
-3 & 1 & 0 & 0 \\
3 & -2 & 1 & 0 \\
-1 & 1 & -1 & 1
\end{array}\right)\left(\begin{array}{llll}
1 & 3 & 3 & 1 \\
0 & 1 & 3 & 3 \\
0 & 0 & 1 & 3 \\
0 & 0 & 0 & 1
\end{array}\right)\left(\begin{array}{llll}
1 & 0 & 0 & 0 \\
3 & 1 & 0 & 0 \\
3 & 2 & 1 & 0 \\
1 & 1 & 1 & 1
\end{array}\right)
$$

Theorem 6.3.

$$
G_{n}^{\beta} x^{p}=\sum_{m=0}^{n}\left(\begin{array}{c}
-n \beta+p \\
m
\end{array}\right)\left(\begin{array}{c}
n \beta+n-p \\
n-m
\end{array}\right) x^{m} .
$$

Proof. We will use the factorial representation of binomial coefficients. Then

$$
\begin{gathered}
\left((1+x)^{n \beta}, x\right)^{T} V_{n} x^{p}=\sum_{i=0}^{n}\left(\begin{array}{c}
n \beta+n-p \\
n-i
\end{array}\right) x^{i}, \\
{[m, \rightarrow] V_{n}^{-1}=\sum_{i=0}^{m}\left(\begin{array}{c}
m-n-1 \\
m-i
\end{array}\right) x^{i}=\sum_{i=0}^{m}(-1)^{m-i}\left(\begin{array}{c}
n-i \\
m-i
\end{array}\right) x^{i},} \\
{\left[x^{m}\right] V_{n}^{-1}\left((1+x)^{n \beta}, x\right)^{T} V_{n} x^{p}=} \\
=\sum_{i=0}^{m}(-1)^{m-i}\left(\begin{array}{c}
n-i \\
m-i
\end{array}\right)\left(\begin{array}{c}
n \beta+n-p \\
n-i
\end{array}\right) \frac{(n \beta+m-p) !}{(n \beta+m-p) !}= \\
=\left(\begin{array}{c}
n \beta+n-p \\
n-m+n-p \\
m-m
\end{array}\right)(-1)^{m}\left(\begin{array}{c}
n \beta+m-p-1 \\
m
\end{array}\right)=\left(\begin{array}{c}
n \beta+n-p \\
n-m
\end{array}\right)\left(\begin{array}{c}
-n \beta+p \\
m
\end{array}\right) .
\end{gathered}
$$

We introduce the matrices $X_{n}=V_{n}^{-1}(x, x)^{T} V_{n}$. We find:

$$
X_{n} x^{0}=\frac{1-x-(1-x)^{n+1}}{x}, \quad X_{n} x^{p}=x^{p-1}(1-x) .
$$


Then

$$
G_{n}^{\beta}=\left(I_{n}+X_{n}\right)^{n \beta}=\sum_{m=0}^{n}\left(\begin{array}{c}
n \beta \\
m
\end{array}\right) X_{n}^{m} .
$$

For example,

$$
\begin{gathered}
G_{3}=I_{3}+3\left(\begin{array}{cccc}
3 & 1 & 0 & 0 \\
-6 & -1 & 1 & 0 \\
4 & 0 & -1 & 1 \\
-1 & 0 & 0 & -1
\end{array}\right)+3\left(\begin{array}{cccc}
3 & 2 & 1 & 0 \\
-8 & -5 & -2 & 1 \\
7 & 4 & 1 & -2 \\
-2 & -1 & 0 & 1
\end{array}\right)+\left(\begin{array}{cccc}
1 & 1 & 1 & 1 \\
-3 & -3 & -3 & -3 \\
3 & 3 & 3 & 3 \\
-1 & -1 & -1 & -1
\end{array}\right), \\
G_{3}^{-1}=I_{3}-3\left(\begin{array}{cccc}
3 & 1 & 0 & 0 \\
-6 & -1 & 1 & 0 \\
4 & 0 & -1 & 1 \\
-1 & 0 & 0 & -1
\end{array}\right)+6\left(\begin{array}{cccc}
3 & 2 & 1 & 0 \\
-8 & -5 & -2 & 1 \\
7 & 4 & 1 & -2 \\
-2 & -1 & 0 & 1
\end{array}\right)-10\left(\begin{array}{cccc}
1 & 1 & 1 & 1 \\
-3 & -3 & -3 & -3 \\
3 & 3 & 3 & 3 \\
-1 & -1 & -1 & -1
\end{array}\right) .
\end{gathered}
$$

Thus, $I_{n}+X_{n}=G_{n}^{1 / n}=U_{n} E U_{n}^{-1}$. For example,

$$
G_{2}^{1 / 2}=\left(\begin{array}{ccc}
3 & 1 & 0 \\
-3 & 0 & 1 \\
1 & 0 & 0
\end{array}\right), \quad G_{3}^{1 / 3}\left(\begin{array}{cccc}
4 & 1 & 0 & 0 \\
-6 & 0 & 1 & 0 \\
4 & 0 & 0 & 1 \\
-1 & 0 & 0 & 0
\end{array}\right), \quad G_{4}^{1 / 4}=\left(\begin{array}{ccccc}
5 & 1 & 0 & 0 & 0 \\
-10 & 0 & 1 & 0 & 0 \\
10 & 0 & 0 & 1 & 0 \\
-5 & 0 & 0 & 0 & 1 \\
1 & 0 & 0 & 0 & 0
\end{array}\right)
$$

Note the identities that follow from the Theorem 2.4.

$$
\begin{gathered}
\left((1-x)^{-m}, x\right) G_{n}^{\beta}\left((1-x)^{m}, x\right) I_{n-m}=G_{n-m}^{\frac{n \beta}{n-m}} . \\
\left((1-x)^{-m}, x\right) G_{n}^{1 / n}\left((1-x)^{m}, x\right) I_{n-m}=G_{n-m}^{1 /(n-m)} .
\end{gathered}
$$

Example 6.1. Let ${ }_{(\beta)} a(x)$ is the generalized binomial series. Then

$$
\begin{array}{cl}
{\left[P_{n}(x)\right](1, x a(x))=x^{n},} & {\left[P_{n}(x)\right]\left(1, x_{(1)} a(x)\right)=x,} \\
{\left[P_{n}(x)\right](a(x), x a(x))=x^{n-1},} & {\left[P_{n}(x)\right]{\left({ }_{(1)} a(x), x_{(1)} a(x)\right)=1 .} a(x)}
\end{array}
$$

Hence,

$$
\begin{gathered}
G_{n}^{\beta} x^{n}=\left[P_{n}(x)\right]\left(1+x\left(\log _{(\beta)} a^{\beta}(x)\right)^{\prime}, x_{(\beta)} a(x)\right), \\
G_{n}^{\beta} x=\left[P_{n}(x)\right]\left(1+x\left(\log _{(\beta+1)} a^{\beta}(x)\right)^{\prime}, x_{(\beta+1)} a(x)\right), \\
\left.G_{n}^{\beta} x^{n-1}=\left[P_{n}(x)\right]{ }_{\left({ }_{(\beta)}\right.} a(x)\left(1+x\left(\log _{(\beta)} a^{\beta}(x)\right)^{\prime}\right), x_{(\beta)} a(x)\right), \\
\left.G_{n}^{\beta} x^{0}=\left[P_{n}(x)\right]{ }_{\left({ }_{(\beta+1)} a(x)\right.}\left(1+x\left(\log _{(\beta+1)} a^{\beta}(x)\right)^{\prime}\right), x_{(\beta+1)} a(x)\right) .
\end{gathered}
$$

Since $G_{n}^{\beta} x^{0}=G_{n}^{\beta+1} x^{n}$, then the identity is manifested here:

$$
{ }_{(\beta)} a(x)\left(1+x\left(\log _{(\beta)} a^{\beta-1}(x)\right)^{\prime}\right)=1+x\left(\log _{(\beta)} a^{\beta}(x)\right)^{\prime} .
$$

To match the identities $G_{n}^{-\beta} x^{0}=J_{n} G_{n}^{\beta} x^{n}, G_{n}^{-\beta} x=J_{n} G_{n}^{\beta} x^{n-1}$ with the definition of polynomials $(-1)^{n} J_{n} g_{n}(x)$, we use the identity ${ }_{(1-\beta)} a(x)={ }_{(\beta)} a^{-1}(-x)$. 


\section{Polynomials ${ }_{(\beta)} h_{n}(x)$.}

Denote

$$
\left[P_{n}(x)\right]\left(b\left(x_{(\beta)} a^{\beta}(x)\right)\left(1+x\left(\log (\beta) a^{\beta}(x)\right)^{\prime}\right), x_{(\beta)} a(x)\right)_{E}={ }_{(\beta)} h_{n}(x) .
$$

We introduce the matrices $H_{n}^{\beta}=F_{n} E^{n \beta} F_{n}^{-1}$. Then $H_{n}^{\beta} h_{n}(x)={ }_{(\beta)} h_{n}(x)$. For example,

$$
H_{1}=\frac{1}{2}\left(\begin{array}{cc}
3 & 1 \\
-1 & 1
\end{array}\right), \quad H_{2}=\frac{1}{6}\left(\begin{array}{ccc}
15 & 5 & 1 \\
-12 & 2 & 4 \\
3 & -1 & 1
\end{array}\right), \quad H_{3}=\frac{1}{20}\left(\begin{array}{cccc}
84 & 28 & 7 & 1 \\
-108 & -4 & 15 & 9 \\
54 & -6 & -1 & 9 \\
-10 & 2 & -1 & 1
\end{array}\right) \text {. }
$$

Sum of elements of each column of the matrix $H_{n}^{\beta}$ is equal to one for the same reason as for the matrix $G_{n}^{\beta}$.

\section{Theorem 7.1.}

$$
H_{n}^{-\beta}=J_{n} H_{n}^{\beta} J_{n}
$$

Proof. By the Theorem 3.1.

$$
J_{n} F_{n} E^{n \beta} F_{n}^{-1} J_{n}=F_{n} E^{n+1}(1,-x) E^{n \beta} E^{n+1}(1,-x) F_{n}^{-1}=F_{n} E^{-n \beta} F_{n}^{-1} .
$$

Matrix $H_{n}^{\beta}$ can be represented as

$$
H_{n}^{\beta}=S_{n} G_{n}^{\beta} S_{n}^{-1}=V_{n}^{-1} C_{n}\left((1+x)^{n \beta}, x\right)^{T} C_{n}^{-1} V_{n} .
$$

For example,

$$
H_{3}=\left(\begin{array}{cccc}
1 & 0 & 0 & 0 \\
-3 & 1 & 0 & 0 \\
3 & -2 & 1 & 0 \\
-1 & 1 & -1 & 1
\end{array}\right)\left(\begin{array}{cccc}
1 & 0 & 0 & 0 \\
0 & 4 & 0 & 0 \\
0 & 0 & 10 & 0 \\
0 & 0 & 0 & 20
\end{array}\right)\left(\begin{array}{cccc}
1 & 3 & 3 & 1 \\
0 & 1 & 3 & 3 \\
0 & 0 & 1 & 3 \\
0 & 0 & 0 & 1
\end{array}\right)\left(\begin{array}{cccc}
1 & 0 & 0 & 0 \\
0 & \frac{1}{4} & 0 & 0 \\
0 & 0 & \frac{1}{10} & 0 \\
0 & 0 & 0 & \frac{1}{20}
\end{array}\right)\left(\begin{array}{cccc}
1 & 0 & 0 & 0 \\
3 & 1 & 0 & 0 \\
3 & 2 & 1 & 0 \\
1 & 1 & 1 & 1
\end{array}\right) .
$$

Denote

$$
t_{n}(\varphi \mid \beta, x)=\sum_{m=0}^{n}\left(\begin{array}{c}
\varphi \\
m
\end{array}\right)\left(\begin{array}{c}
\beta \\
n-m
\end{array}\right) x^{m}
$$

Theorem7. 2.

$$
H_{n}^{\beta} x^{p}=\sum_{m=p}^{n}\left(\begin{array}{c}
n+m \\
m
\end{array}\right)^{-1}\left(\begin{array}{c}
n-p \\
n-m
\end{array}\right)(1-x)^{n-m} t_{m}(-n \beta+n+m \mid n \beta, x) .
$$

Proof. If $c_{p}(x)$ is a polynomial of degree $p \leq n$, then $V_{n}^{-1} c_{p}(x)=(1-x)^{n-p} V_{p}^{-1} c_{p}(x)$. Then

$$
\begin{gathered}
\frac{1}{n !}\left[x^{m}\right] V_{p}^{-1} C_{n}\left((1+x)^{n \beta}, x\right)^{T} x^{p}= \\
=\sum_{i=0}^{m}(-1)^{m-i}\left(\begin{array}{c}
p-i \\
m-i
\end{array}\right)\left(\begin{array}{c}
n \beta \\
p-i
\end{array}\right)\left(\begin{array}{c}
n+i \\
i
\end{array}\right) \frac{(n \beta+m-p) !}{(n \beta+m-p) !}= \\
=\left(\begin{array}{c}
n \beta \\
p-m
\end{array}\right) \sum_{i=0}^{m}(-1)^{m-i}\left(\begin{array}{c}
n+i \\
i
\end{array}\right)\left(\begin{array}{c}
n \beta+m-p \\
m-i
\end{array}\right)= \\
=\left(\begin{array}{c}
n \beta \\
p-m
\end{array}\right)(-1)^{m}\left(\begin{array}{c}
n \beta+m-p-n-1 \\
m
\end{array}\right)=\left(\begin{array}{c}
n \beta \\
p-m
\end{array}\right)\left(\begin{array}{c}
-n \beta+n+p \\
m
\end{array}\right),
\end{gathered}
$$




$$
V_{n}^{-1} C_{n}\left((1+x)^{n \beta}, x\right)^{T} x^{p}=n !(1-x)^{n-p} t_{p}(-n \beta+n+p \mid n \beta, x) .
$$

It remains to add that

$$
C_{n}^{-1} V_{n} x^{p}=\frac{1}{n !} \sum_{m=p}^{n}\left(\begin{array}{c}
n+m \\
m
\end{array}\right)^{-1}\left(\begin{array}{c}
n-p \\
n-m
\end{array}\right) x^{m} .
$$

In particular,

$$
\begin{gathered}
H_{n}^{\beta} x^{n}=\left(\begin{array}{c}
2 n \\
n
\end{array}\right)^{-1} \sum_{m=0}^{n}\left(\begin{array}{c}
-n \beta+2 n \\
m
\end{array}\right)\left(\begin{array}{c}
n \beta \\
n-m
\end{array}\right) x^{m}, \\
H_{n}^{\beta} x^{0}=\left(\begin{array}{c}
2 n \\
n
\end{array}\right)^{-1} \sum_{m=0}^{n}\left(\begin{array}{c}
-n \beta \\
m
\end{array}\right)\left(\begin{array}{c}
n \beta+2 n \\
n-m
\end{array}\right) x^{m} .
\end{gathered}
$$

Example 7.1. Let ${ }_{(\beta)} a(x)$ is the generalized binomial series. Then, considering that $(1, x a(x))^{-1}=\left(1, x_{(2)} a(-x)\right)$,

$$
\begin{gathered}
{\left[P_{n}(x)\right](1, x a(x))_{E}=S_{n} x^{n}=\frac{(2 n) !}{n !} x^{n}, \quad\left[P_{n}(x)\right]\left(1, x_{(2)} a(x)\right)_{E}=\frac{(2 n) !}{n !} x,} \\
{\left[P_{n}(x)\right]\left((x a(x))^{\prime}, x a(x)\right)_{E}=\frac{(2 n) !}{n !} x^{n-1}, \quad\left[P_{n}(x)\right]\left(\left(x_{(2)} a(x)\right)^{\prime}, x_{(2)} a(x)\right)_{E}=\frac{(2 n) !}{n !},} \\
{\left[P_{n}(x)\right](a(x), x a(x))_{E}=S_{n} x^{n-1}=\frac{(2 n) !}{n ! 2}(1+x) x^{n-1},} \\
{\left[P_{n}(x)\right]\left(1+x\left(\log _{(2)} a(x)\right)^{\prime}, x_{(2)} a(x)\right)_{E}=\frac{(2 n) !}{n ! 2}(1+x) .}
\end{gathered}
$$

Hence,

$$
\begin{gathered}
\frac{(2 n) !}{n !} H_{n}^{\beta} x^{n}=\left[P_{n}(x)\right]\left(1+x\left(\log _{(\beta)} a^{\beta}(x)\right)^{\prime}, x_{(\beta)} a(x)\right)_{E} \\
\frac{(2 n) !}{n !} H_{n}^{\beta} x^{0}=\frac{(2 n) !}{n !} H_{n}^{\beta+2} x^{n}=\left[P_{n}(x)\right]\left(1+x\left(\log _{(\beta+2)} a^{\beta+2}(x)\right)^{\prime}, x_{(\beta+2)} a(x)\right)_{E} \\
\frac{(2 n) !}{n !} H_{n}^{\beta} x=\left[P_{n}(x)\right]\left(1+x\left(\log _{(\beta+2)} a^{\beta}(x)\right)^{\prime}, x_{(\beta+2)} a(x)\right)_{E} \\
\frac{(2 n) !}{n !} H_{n}^{\beta} x^{n-1}=\left[P_{n}(x)\right]\left({ }_{(\beta)} a(x)\left(1+x\left(\log _{(\beta)} a^{\beta+1}(x)\right)^{\prime}\right), x_{(\beta)} a(x)\right)_{E} \\
\frac{(2 n) !}{n ! 2} H_{n}^{\beta}(1+x) x^{n-1}=\left[P_{n}(x)\right]\left({ }_{(\beta)} a(x)\left(1+x\left(\log _{(\beta)} a^{\beta}(x)\right)^{\prime}\right), x_{(\beta)} a(x)\right)_{E} \\
\frac{(2 n) !}{n ! 2} H_{n}^{\beta}(1+x)=\left[P_{n}(x)\right]\left(1+x\left(\log _{(\beta+2)} a^{\beta+1}(x)\right)^{\prime}, x_{(\beta+2)} a(x)\right)_{E}
\end{gathered}
$$

Note that

$$
\begin{gathered}
\left(\begin{array}{c}
2 n-1 \\
n-1
\end{array}\right) H_{n}^{\beta}(1+x) x^{n-1}=(1-x) \sum_{m=0}^{n-1}\left(\begin{array}{c}
-n \beta+2 n-1 \\
m
\end{array}\right)\left(\begin{array}{c}
n \beta \\
n-1-m
\end{array}\right) x^{m}+ \\
+\sum_{m=0}^{n}\left(\begin{array}{c}
-n \beta+2 n \\
m
\end{array}\right)\left(\begin{array}{c}
n \beta \\
n-m
\end{array}\right) x^{m}=\sum_{m=0}^{n}\left(\begin{array}{c}
-n \beta+2 n-1 \\
m
\end{array}\right)\left(\begin{array}{c}
n \beta+1 \\
n-m
\end{array}\right) x^{m}, \\
\left(\begin{array}{c}
2 n-1 \\
n-1
\end{array}\right) H_{n}^{\beta}(1+x)=\sum_{m=0}^{n}\left(\begin{array}{c}
-n \beta+1 \\
m
\end{array}\right)\left(\begin{array}{c}
n \beta+2 n-1 \\
n-m
\end{array}\right) x^{m} .
\end{gathered}
$$

To match the identities $H_{n}^{-\beta} x^{0}=J_{n} H_{n}^{\beta} x^{n}, H_{n}^{-\beta} x=J_{n} H_{n}^{\beta} x^{n-1}$ with the definition of polynomials $(-1)^{n} J_{n} h_{n}(x)$, we use the identities $\left(1, x_{(\beta)} a(x)\right)^{-1}=\left(1, x_{(\beta-1)} a^{-1}(x)\right)$, ${ }_{(\beta-1)} a^{-1}(-x)={ }_{(2-\beta)} a(x)$. 


\section{Matrices $\tilde{U}_{n}, \tilde{F}_{n}$}

Let, as before, $\left[P_{n}(x)\right](1, x a(x))=\alpha_{n}(x),[n, \rightarrow](1, \log a(x))_{E}=u_{n}(x)$. For the sequence of polynomials $c_{n}(x)$ such that $c_{0}(0)=1, c_{n}(0)=0$ we denote: $\tilde{c}_{0}(x)=1$, $\tilde{c}_{n}(x)=(1 / x) c_{n}(x)$. We introduce the matrices $\tilde{U}_{n}, n>0$ :

$$
\begin{gathered}
\tilde{U}_{n}=(x, x)^{T} U_{n}(x, x), \quad \tilde{U}_{n}^{-1}=(x, x)^{T} U_{n}^{-1}(x, x), \\
\tilde{U}_{n} x^{p}=\frac{1}{n !}(1-x)^{n-1-p} \tilde{A}_{p+1}(x), \quad \tilde{U}_{n}^{-1} x^{p}=(x-1)_{p}[x+1]_{n-p-1}, \quad 0 \leq p<n .
\end{gathered}
$$

For example,

$$
\tilde{U}_{4}=\frac{1}{4 !}\left(\begin{array}{cccc}
1 & 1 & 1 & 1 \\
-3 & -1 & 3 & 11 \\
3 & -1 & -3 & 11 \\
-1 & 1 & -1 & 1
\end{array}\right), \quad \tilde{U}_{4}^{-1}=\left(\begin{array}{cccc}
6 & -2 & 2 & -6 \\
11 & -1 & -1 & 11 \\
6 & 2 & -2 & -6 \\
1 & 1 & 1 & 1
\end{array}\right)
$$

Then $\tilde{U}_{n} \tilde{u}_{n}(x)=\tilde{\alpha}_{n}(x)$. Since

$$
\frac{\tilde{\alpha}_{n}(x)}{(1-x)^{n+1}}=\sum_{m=0}^{\infty} \frac{u_{n}(m+1)}{n !} x^{m}, \quad \tilde{\alpha}_{n}(x)=U_{n} E u_{n}(x)
$$

then the matrices $\tilde{U}_{n}, \tilde{U}_{n}^{-1}$ can be represented as

$$
\tilde{U}_{n}=U_{n} E(x, x) I_{n-1}, \quad \tilde{U}_{n}^{-1}=(x, x)^{T} E^{-1} U_{n}^{-1} I_{n-1} .
$$

We introduce the matrices $\tilde{J}_{n}=J_{n-1}, \tilde{I}_{n}=I_{n-1}, \tilde{V}_{n}=V_{n-1}$. Then $\tilde{U}_{n}^{-1} \tilde{V}_{n}^{-1} x^{p}=\frac{n !}{(p+1) !} \sum_{m=0}^{p} s(p+1, m+1) x^{m}, \quad \tilde{V}_{n} \tilde{U}_{n} x^{p}=\frac{1}{n !} \sum_{m=0}^{p} m ! S(p+1, m+1) x^{m}$.

For example, $\tilde{U}_{4}^{-1} \tilde{V}_{4}^{-1}, \tilde{V}_{4} \tilde{U}_{4}$ :

$$
4 !\left(\begin{array}{cccc}
1 & -1 & 2 & -6 \\
0 & 1 & -3 & 11 \\
0 & 0 & 1 & -6 \\
0 & 0 & 0 & 1
\end{array}\right)\left(\begin{array}{cccc}
1 & 0 & 0 & 0 \\
0 & \frac{1}{2} & 0 & 0 \\
0 & 0 & \frac{1}{3 !} & 0 \\
0 & 0 & 0 & \frac{1}{4 !}
\end{array}\right), \quad \frac{1}{4 !}\left(\begin{array}{cccc}
1 & 0 & 0 & 0 \\
0 & 2 & 0 & 0 \\
0 & 0 & 3 ! & 0 \\
0 & 0 & 0 & 4 !
\end{array}\right)\left(\begin{array}{cccc}
1 & 1 & 1 & 1 \\
0 & 1 & 3 & 7 \\
0 & 0 & 1 & 6 \\
0 & 0 & 0 & 1
\end{array}\right) .
$$

Theorem 8.1.

$$
\tilde{U}_{n}(1,-x) \tilde{U}_{n}^{-1}=(-1)^{n-1} \tilde{J}_{n}
$$

Proof.

$$
(1,-x)(x-1)_{p}[x+1]_{n-p-1}=(-x-1)_{p}[-x+1]_{n-p-1}=(-1)^{n-1}(x-1)_{n-p-1}[x+1]_{p} .
$$

Example 8.1. This example illustrates the advantage of the matrices $\tilde{U}_{n}$ over the matrices $U_{n}$ in certain cases. Denote

$$
\left[P_{n}(x)\right]\left(1, x a^{m}(x)\right)=\alpha_{n}^{(m)}(x), \quad W_{(n, m)}=\tilde{U}_{n}(m, m x) \tilde{U}_{n}^{-1} .
$$

Then $W_{(n, m)} \tilde{\alpha}_{n}(x)=\tilde{\alpha}_{n}^{(m)}(x)$. We build the matrix $(a(x), x)_{m}$ by the rule $[n, \rightarrow](a(x), x)_{m}=$ $[m n+m-1, \rightarrow](a(x), x)$. For example,

$$
(a(x), x)_{2}=\left(\begin{array}{ccccc}
a_{1} & a_{0} & 0 & 0 & \cdots \\
a_{3} & a_{2} & a_{1} & a_{0} & \cdots \\
a_{5} & a_{4} & a_{3} & a_{2} & \cdots \\
a_{7} & a_{6} & a_{5} & a_{4} & \cdots \\
\vdots & \vdots & \vdots & \vdots & \ddots
\end{array}\right), \quad(a(x), x)_{3}=\left(\begin{array}{ccccc}
a_{2} & a_{1} & a_{0} & 0 & \cdots \\
a_{5} & a_{4} & a_{3} & a_{2} & \cdots \\
a_{8} & a_{7} & a_{6} & a_{5} & \cdots \\
a_{11} & a_{10} & a_{9} & a_{8} & \cdots \\
\vdots & \vdots & \vdots & \vdots & \ddots
\end{array}\right) .
$$


Theorem 8.2.

$$
W_{(n, m)}=\left(w_{m}^{n+1}(x), x\right)_{m} \tilde{I}_{n}, \quad w_{m}^{n+1}(x)=\left(\frac{1-x^{m}}{1-x}\right)^{n+1} .
$$

Proof. Since

$$
\begin{gathered}
{\left[x^{p}\right] \frac{\tilde{\alpha}_{n}^{(m)}(x)}{(1-x)^{n+1}}=\left[x^{m p+m-1}\right] \frac{\tilde{\alpha}_{n}(x)}{(1-x)^{n+1}}, \quad \frac{\tilde{\alpha}_{n}(x)}{(1-x)^{n+1}}=\frac{w_{m}^{n+1}(x) \tilde{\alpha}_{n}(x)}{\left(1-x^{m}\right)^{n+1}}=\sum_{r=0}^{m-1} \frac{x^{r} c_{r}(x)}{\left(1-x^{m}\right)^{n+1}},} \\
c_{r}(x)=\sum_{p=0}^{\infty}\left(\left[x^{m p+r}\right] w_{m}^{n+1}(x) \tilde{\alpha}_{n}(x)\right) x^{m p},
\end{gathered}
$$

then

$$
\begin{gathered}
\frac{\tilde{\alpha}_{n}^{(m)}\left(x^{m}\right)}{\left(1-x^{m}\right)^{n+1}}=\frac{c_{m-1}(x)}{\left(1-x^{m}\right)^{n+1}}, \quad\left[x^{p}\right] \tilde{\alpha}_{n}^{(m)}(x)=\left[x^{m p+m-1}\right] w_{m}^{n+1}(x) \tilde{\alpha}_{n}(x), \\
\tilde{\alpha}_{n}^{(m)}(x)=\left(w_{m}^{n+1}(x), x\right)_{m} \tilde{\alpha}_{n}(x) .
\end{gathered}
$$

For example, $W_{(1,2)}=(2), W_{(1,3)}=(3), W_{(1,4)}=(4)$,

$$
\begin{gathered}
W_{(2,2)}=\left(\begin{array}{ll}
3 & 1 \\
1 & 3
\end{array}\right), \\
W_{(3,2)}=\left(\begin{array}{lll}
4 & 1 & 0 \\
4 & 6 & 4 \\
0 & 1 & 4
\end{array}\right),
\end{gathered}
$$

Since $\alpha_{n}^{(m)}(1)=m^{n} \alpha_{n}(1)$, then the sum of elements of each column of the matrix $W_{(n, m)}$ is equal to $m^{n}$. Note the identities

$$
\begin{gathered}
W_{(n, m)} \tilde{A}_{n}(x)=m^{n} \tilde{A}_{n}(x), \quad W_{(n, m)} \tilde{J}_{n}=\tilde{J}_{n} W_{(n, m)}, \quad W_{(n, m)} W_{(n, p)}=W_{(n, m p)}, \\
\left((1-x)^{-p}, x\right) W_{(n, m)}\left((1-x)^{p}, x\right) \tilde{I}_{n-p}=W_{(n-p, m)} .
\end{gathered}
$$

For example,

$$
\begin{gathered}
\left(\begin{array}{lll}
4 & 1 & 0 \\
4 & 6 & 4 \\
0 & 1 & 4
\end{array}\right)\left(\begin{array}{l}
1 \\
4 \\
1
\end{array}\right)=8\left(\begin{array}{l}
1 \\
4 \\
1
\end{array}\right), \quad\left(\begin{array}{ccc}
10 & 4 & 1 \\
16 & 19 & 16 \\
1 & 4 & 10
\end{array}\right)\left(\begin{array}{l}
1 \\
4 \\
1
\end{array}\right)=27\left(\begin{array}{l}
1 \\
4 \\
1
\end{array}\right), \\
\left(\begin{array}{lll}
4 & 1 & 0 \\
4 & 6 & 4 \\
0 & 1 & 4
\end{array}\right)\left(\begin{array}{lll}
4 & 1 & 0 \\
4 & 6 & 4 \\
0 & 1 & 4
\end{array}\right)=\left(\begin{array}{ccc}
20 & 10 & 4 \\
40 & 44 & 40 \\
4 & 10 & 20
\end{array}\right), \\
\left(\begin{array}{lll}
1 & 0 & 0 \\
1 & 1 & 0 \\
1 & 1 & 1
\end{array}\right)\left(\begin{array}{lll}
4 & 1 & 0 \\
4 & 6 & 4 \\
0 & 1 & 4
\end{array}\right)\left(\begin{array}{cc}
1 & 0 \\
-1 & 1 \\
0 & -1
\end{array}\right)=\left(\begin{array}{ll}
3 & 1 \\
1 & 3
\end{array}\right)
\end{gathered}
$$




$$
\left(\begin{array}{lll}
1 & 0 & 0 \\
2 & 1 & 0 \\
3 & 2 & 1
\end{array}\right)\left(\begin{array}{lll}
4 & 1 & 0 \\
4 & 6 & 4 \\
0 & 1 & 4
\end{array}\right)\left(\begin{array}{c}
1 \\
-2 \\
1
\end{array}\right)=\left(\begin{array}{ll}
1 & 0 \\
1 & 1
\end{array}\right)\left(\begin{array}{ll}
3 & 1 \\
1 & 3
\end{array}\right)\left(\begin{array}{c}
1 \\
-1
\end{array}\right)=(2)
$$

Since $(1, a(x)-1)\left(1,(1+x)^{m}-1\right)=\left(1, a^{m}(x)-1\right)$, then the matrix $W_{(n, m)}$ can be represented as

$$
W_{(n, m)}=\tilde{V}_{n}^{-1}\left(\frac{(1+x)^{m}-1}{x},(1+x)^{m}-1\right)^{T} \tilde{V}_{n}
$$

For example,

$$
\begin{aligned}
\left(\begin{array}{lll}
4 & 1 & 0 \\
4 & 6 & 4 \\
0 & 1 & 4
\end{array}\right) & =\frac{1}{3 !}\left(\begin{array}{ccc}
1 & 1 & 1 \\
-2 & 0 & 4 \\
1 & -1 & 1
\end{array}\right)\left(\begin{array}{lll}
2 & 0 & 0 \\
0 & 4 & 0 \\
0 & 0 & 8
\end{array}\right)\left(\begin{array}{ccc}
2 & -1 & 2 \\
3 & 0 & -3 \\
1 & 1 & 1
\end{array}\right)= \\
& =\left(\begin{array}{ccc}
1 & 0 & 0 \\
-2 & 1 & 0 \\
1 & -1 & 1
\end{array}\right)\left(\begin{array}{lll}
2 & 1 & 0 \\
0 & 4 & 4 \\
0 & 0 & 8
\end{array}\right)\left(\begin{array}{lll}
1 & 0 & 0 \\
2 & 1 & 0 \\
1 & 1 & 1
\end{array}\right) .
\end{aligned}
$$

Matrices $\left(1 / m^{n}\right)\left(W_{(n, m)}\right)^{T}$ are known as amazing matrices [27, p.156]. They find application in various fields of mathematics [28] - [31].

Denote $\left[P_{n}(x)\right](1, x a(x))_{E}=\varphi_{n}(x)$. We introduce the matrices $\tilde{F}_{n}$ :

$$
\begin{gathered}
\tilde{F}_{n}=(x, x)^{T} F_{n}(x, x), \quad \tilde{F}_{n}^{-1}=(x, x)^{T} U_{n}^{-1}(x, x), \\
\tilde{F}_{n} x^{p}=(1-x)^{2 n+1} \sum_{m=0}^{\infty}(m+1)^{p+1}\left(\begin{array}{c}
m+n+1 \\
n
\end{array}\right) x^{m}, \\
\tilde{F}_{n}^{-1} x^{p}=\frac{n !}{(2 n) !}(x-1)_{p}[x+n+1]_{n-p-1}, \quad 0 \leq p<n .
\end{gathered}
$$

For example,

$$
\tilde{F}_{4}=5\left(\begin{array}{cccc}
1 & 1 & 1 & 1 \\
-3 & 3 & 15 & 39 \\
3 & -9 & 9 & 171 \\
-1 & 5 & -25 & 125
\end{array}\right), \quad \tilde{F}_{4}^{-1}=\frac{4 !}{8 !}\left(\begin{array}{cccc}
210 & -30 & 10 & -6 \\
107 & 19 & -13 & 11 \\
18 & 10 & 2 & -6 \\
1 & 1 & 1 & 1
\end{array}\right)
$$

Then $\tilde{F}_{n} \tilde{u}_{n}(x)=\tilde{\varphi}_{n}(x)$. Since (see Example 3.1.)

$$
\frac{\tilde{\varphi}_{n}(x)}{(1-x)^{2 n+1}}=\sum_{m=0}^{\infty}\left(\begin{array}{c}
n+m \\
m
\end{array}\right)(m+n+1) \tilde{u}_{n}(m+1) x^{m}, \quad \tilde{\varphi}_{n}(x)=F_{n} E(x+n) \tilde{u}_{n}(x),
$$

then the matrices $\tilde{F}_{n}, \tilde{F}_{n}^{-1}$ can be represented as

$$
\tilde{F}_{n}=F_{n} E(x+n, x) I_{n-1}=F_{n}(x+n+1, x) E I_{n-1}, \quad \tilde{F}_{n}^{-1}=(x+n, x)^{-1} E^{-1} F_{n}^{-1} I_{n-1} .
$$

Note that since

$$
\begin{gathered}
{\left[x^{n}\right] F_{n} x^{p}(x+n+1)=\left[x^{n}\right] F_{n} E x^{p}(x+n)=0, \quad p<n ;} \\
F_{n} x^{p}=(1-x)^{2 n+1} \sum_{m=0}^{\infty} m^{p}\left(\begin{array}{c}
m+n \\
n
\end{array}\right) x^{m}, \quad F_{n} E I_{n} x^{p}=(1-x)^{2 n+1} \sum_{m=0}^{\infty}(m+1)^{p}\left(\begin{array}{c}
m+n \\
n
\end{array}\right) x^{m},
\end{gathered}
$$

then the identities for the $n$th elements of columns of the matrices $F_{n}, F_{n} E I_{n}$ are manifested here:

$$
\sum_{m=0}^{n}(-1)^{n-m}\left(\begin{array}{c}
2 n+1 \\
n-m
\end{array}\right) m^{p}\left(\begin{array}{c}
m+n \\
n
\end{array}\right)=(-1)^{n+p}(n+1)^{p}
$$




$$
\sum_{m=0}^{n}(-1)^{n-m}\left(\begin{array}{c}
2 n+1 \\
n-m
\end{array}\right)(m+1)^{p}\left(\begin{array}{c}
m+n \\
n
\end{array}\right)=(-1)^{n+p} n^{p}, \quad p \leq n .
$$

Theorem 8.3.

$$
\tilde{F}_{n} E^{n}(1,-x) \tilde{F}_{n}^{-1}=(-1)^{n-1} \tilde{J}_{n}
$$

Proof.

$$
\begin{gathered}
E^{n}(1,-x)(x-1)_{p}[x+n+1]_{n-p-1}=(-x-n-1)_{p}[-x+1]_{n-p-1}= \\
=(-1)^{n-1}(x-1)_{n-p-1}[x+n+1]_{p} . \quad \square
\end{gathered}
$$

We introduce the matrices $\tilde{S}_{n}=\tilde{V}_{n} \tilde{C}_{n} \tilde{V}_{n}, \tilde{C}_{n} x^{p}=((n+p+1) ! /(p+1) !) x^{p}$. Then $\tilde{S}_{n} \tilde{\alpha}_{n}(x)=\tilde{\varphi}_{n}(x)$.

\section{Polynomials ${ }_{(\beta)} \alpha_{n}(x),{ }_{(\beta)} \varphi_{n}(x)$}

Denote $\left[P_{n}(x)\right]\left(1, x_{(\beta)} a(x)\right)={ }_{(\beta)} \alpha_{n}(x)$. We introduce the matrices $A_{n}^{\beta}=\tilde{U}_{n} E^{n \beta} \tilde{U}_{n}^{-1}$. Then $A_{n}^{\beta} \tilde{\alpha}_{n}(x)={ }_{(\beta)} \tilde{\alpha}_{n}(x)$. For example,

$$
A_{2}=\left(\begin{array}{cc}
2 & 1 \\
-1 & 0
\end{array}\right), \quad A_{3}=\left(\begin{array}{ccc}
5 & 5 / 2 & 1 \\
-6 & -2 & 0 \\
2 & 1 / 2 & 0
\end{array}\right), \quad A_{4}=\left(\begin{array}{cccc}
14 & 7 & 3 & 1 \\
-28 & -35 / 3 & -10 / 3 & 0 \\
20 & 22 / 3 & 5 / 3 & 0 \\
-5 & -5 / 3 & -1 / 3 & 0
\end{array}\right)
$$

\section{Theorem 9.1.}

$$
A_{n}^{-\beta}=\tilde{J}_{n} A_{n}^{\beta} \tilde{J}_{n}
$$

Proof. By the Theorem 8.1.

$$
\tilde{J}_{n} \tilde{U}_{n} E^{n \beta} \tilde{U}_{n}^{-1} \tilde{J}_{n}=\tilde{U}_{n}(1,-x) E^{n \beta}(1,-x) \tilde{U}_{n}^{-1}=\tilde{U}_{n} E^{-n \beta} \tilde{U}_{n}^{-1}
$$

Note the identity

$$
\left((1-x)^{-m}, x\right) A_{n}^{\beta}\left((1-x)^{m}, x\right) \tilde{I}_{n-m}=A_{n-m}^{\frac{n \beta}{n-m}} .
$$

Let $D$ is the matrix of derivation operator. We introduce the diagonal matrix $\tilde{D}=D(x, x), \tilde{D} x^{n}=(n+1) x^{n}$.

Theorem 9.2.

$$
A_{n}^{\beta}=\tilde{V}_{n}^{-1} \tilde{D}\left((1+x)^{n \beta}, x\right)^{T} \tilde{D}^{-1} \tilde{V}_{n} .
$$

Proof. Since

$$
\begin{gathered}
n !\left|e^{x}\right| \tilde{D}^{-1} \tilde{V}_{n} \tilde{U}_{n} x^{p}=[p, \rightarrow]\left(e^{x}, e^{x}-1\right)_{E} \\
(1 / n !) \tilde{U}_{n}^{-1} \tilde{V}_{n}^{-1}\left|e^{x}\right|^{-1} \tilde{D} x^{p}=[p, \rightarrow]\left((1+x)^{-1}, \log (1+x)\right)_{E} \\
\left((1+x)^{-1}, \log (1+x)\right)_{E}\left(e^{n \beta}, x\right)_{E}\left(e^{x}, e^{x}-1\right)_{E}=\left((1+x)^{n \beta}, x\right)_{E}
\end{gathered}
$$

then

$$
\tilde{V}_{n} \tilde{U}_{n} E^{n \beta} \tilde{U}_{n}^{-1} \tilde{V}_{n}^{-1}=\tilde{D}\left((1+x)^{n \beta}, x\right)^{T} \tilde{D}^{-1} \tilde{I}_{n} .
$$

For example,

$$
A_{4}=\left(\begin{array}{cccc}
1 & 0 & 0 & 0 \\
-3 & 1 & 0 & 0 \\
3 & -2 & 1 & 0 \\
-1 & 1 & -1 & 1
\end{array}\right)\left(\begin{array}{llll}
1 & 0 & 0 & 0 \\
0 & 2 & 0 & 0 \\
0 & 0 & 3 & 0 \\
0 & 0 & 0 & 4
\end{array}\right)\left(\begin{array}{cccc}
1 & 4 & 6 & 4 \\
0 & 1 & 4 & 6 \\
0 & 0 & 1 & 4 \\
0 & 0 & 0 & 1
\end{array}\right)\left(\begin{array}{cccc}
1 & 0 & 0 & 0 \\
0 & \frac{1}{2} & 0 & 0 \\
0 & 0 & \frac{1}{3} & 0 \\
0 & 0 & 0 & \frac{1}{4}
\end{array}\right)\left(\begin{array}{cccc}
1 & 0 & 0 & 0 \\
3 & 1 & 0 & 0 \\
3 & 2 & 1 & 0 \\
1 & 1 & 1 & 1
\end{array}\right) .
$$


Theorem 9.3.

$$
T_{n}^{\beta} x^{p}=\sum_{m=p}^{n-1} \frac{1}{(m+1)}\left(\begin{array}{c}
n-1-p \\
n-1-m
\end{array}\right)(1-x)^{n-m-1} t_{m}(-n \beta+m+1 \mid n \beta, x) .
$$

Proof. In this case $p=0,1, \ldots, n-1$. Then

$$
\begin{gathered}
{\left[x^{m}\right] \tilde{V}_{p+1}^{-1} \tilde{D}\left((1+x)^{n \beta}, x\right)^{T} x^{p}=} \\
=\sum_{i=0}^{m}(-1)^{m-i}\left(\begin{array}{c}
p-i \\
m-i
\end{array}\right)\left(\begin{array}{c}
n \beta \\
p-i
\end{array}\right)(i+1) \frac{(n \beta+m-p) !}{(n \beta+m-p) !}= \\
=\left(\begin{array}{c}
n \beta \\
p-m
\end{array}\right) \sum_{i=0}^{m}(-1)^{m-i}(i+1)\left(\begin{array}{c}
n \beta+m-p \\
m-i
\end{array}\right)= \\
=\left(\begin{array}{c}
n \beta \\
p-m
\end{array}\right)(-1)^{m}\left(\begin{array}{c}
n \beta+m-p-2 \\
m
\end{array}\right)=\left(\begin{array}{c}
n \beta \\
p-m
\end{array}\right)\left(\begin{array}{c}
-n \beta+p+1 \\
m
\end{array}\right), \\
\tilde{V}_{n}^{-1} \tilde{D}\left((1+x)^{n \beta}, x\right)^{T} x^{p}=(1-x)^{n-p-1} t_{p}(-n \beta+p+1 \mid n \beta, x) . \\
\tilde{D}^{-1} \tilde{V}_{n} x^{p}=\sum_{m=p}^{n-1} \frac{1}{(m+1)}\left(\begin{array}{c}
n-1-p \\
n-1-m
\end{array}\right) x^{m} .
\end{gathered}
$$

In particular,

$A_{n}^{\beta} x^{n-1}=\frac{1}{n} \sum_{m=0}^{n-1}\left(\begin{array}{c}n(1-\beta) \\ m\end{array}\right)\left(\begin{array}{c}n \beta \\ n-1-m\end{array}\right) x^{m}, \quad A_{n}^{\beta} x^{0}=\frac{1}{n} \sum_{m=0}^{n-1}\left(\begin{array}{c}-n \beta \\ m\end{array}\right)\left(\begin{array}{c}n(1+\beta) \\ n-1-m\end{array}\right) x^{m}$,

that corresponds to the formula (2).

Denote $\left[P_{n}(x)\right]\left(1, x_{(\beta)} a(x)\right)_{E}={ }_{(\beta)} \varphi_{n}(x)$. We introduce the matrices $T_{n}^{\beta}=\tilde{F}_{n} E^{n \beta} \tilde{F}_{n}^{-1}$. Then $T_{n}^{\beta} \tilde{\varphi}_{n}(x)={ }_{(\beta)} \tilde{\varphi}_{n}(x)$. For example,

$$
T_{2}=\frac{1}{2}\left(\begin{array}{cc}
3 & 1 \\
-1 & 1
\end{array}\right), \quad T_{3}=\frac{1}{5}\left(\begin{array}{ccc}
12 & 4 & 1 \\
-9 & 2 & 3 \\
2 & -1 & 1
\end{array}\right), \quad T_{4}=\frac{1}{14}\left(\begin{array}{cccc}
55 & 55 / 3 & 5 & 1 \\
-66 & 0 & 10 & 6 \\
30 & -6 & 0 & 6 \\
-5 & 5 / 3 & -1 & 1
\end{array}\right) .
$$

Theorem 9.4.

$$
T_{n}^{-\beta}=\tilde{J}_{n} T_{n}^{\beta} \tilde{J}_{n}
$$

Proof. By the Theorem 8.3.

$$
\tilde{J}_{n} \tilde{F}_{n} E^{n \beta} \tilde{F}_{n}^{-1} \tilde{J}_{n}=\tilde{F}_{n} E^{n}(1,-x) E^{n \beta} E^{n}(1,-x) \tilde{F}_{n}^{-1}=\tilde{F}_{n} E^{-n \beta} \tilde{F}_{n}^{-1} .
$$

Matrix $T_{n}^{\beta}$ can be represented as

$$
\begin{gathered}
T_{n}^{\beta}=\tilde{S}_{n} A_{n}^{\beta} \tilde{S}_{n}^{-1}=\tilde{V}_{n}^{-1} \tilde{C}_{n} \tilde{D}\left((1+x)^{n \beta}, x\right)^{T} \tilde{D}^{-1} \tilde{C}_{n}^{-1} \tilde{V}_{n}, \\
\tilde{C}_{n} \tilde{D} x^{p}=(n+1) !\left(\begin{array}{c}
n+1+p \\
p
\end{array}\right) x^{p} .
\end{gathered}
$$

For example,

$$
T_{4}=\left(\begin{array}{cccc}
1 & 0 & 0 & 0 \\
-3 & 1 & 0 & 0 \\
3 & -2 & 1 & 0 \\
-1 & 1 & -1 & 1
\end{array}\right)\left(\begin{array}{cccc}
1 & 0 & 0 & 0 \\
0 & 6 & 0 & 0 \\
0 & 0 & 21 & 0 \\
0 & 0 & 0 & 56
\end{array}\right)\left(\begin{array}{cccc}
1 & 4 & 6 & 4 \\
0 & 1 & 4 & 6 \\
0 & 0 & 1 & 4 \\
0 & 0 & 0 & 1
\end{array}\right)\left(\begin{array}{cccc}
1 & 0 & 0 & 0 \\
0 & \frac{1}{6} & 0 & 0 \\
0 & 0 & \frac{1}{21} & 0 \\
0 & 0 & 0 & \frac{1}{56}
\end{array}\right)\left(\begin{array}{cccc}
1 & 0 & 0 & 0 \\
3 & 1 & 0 & 0 \\
3 & 2 & 1 & 0 \\
1 & 1 & 1 & 1
\end{array}\right) .
$$




\section{Theorem 9.5.}

$$
T_{n}^{\beta} x^{p}=\sum_{m=p}^{n-1}\left(\begin{array}{c}
n+1+m \\
m
\end{array}\right)^{-1}\left(\begin{array}{c}
n-1-p \\
n-1-m
\end{array}\right)(1-x)^{n-m-1} t_{m}(-n \beta+n+m+1 \mid n \beta, x) .
$$

\section{Proof.}

$$
\begin{gathered}
\frac{1}{(n+1) !}\left[x^{m}\right] \tilde{V}_{p+1}^{-1} \tilde{C}_{n} \tilde{D}\left((1+x)^{n \beta}, x\right)^{T} x^{p}= \\
=\sum_{i=0}^{m}(-1)^{m-i}\left(\begin{array}{c}
p-i \\
m-i
\end{array}\right)\left(\begin{array}{c}
n \beta \\
p-i
\end{array}\right)\left(\begin{array}{c}
n+1+i \\
i
\end{array}\right) \frac{(n \beta+m-p) !}{(n \beta+m-p) !}= \\
=\left(\begin{array}{c}
n \beta \\
p-m
\end{array}\right) \sum_{i=0}^{m}(-1)^{m-i}\left(\begin{array}{c}
n+1+i \\
i
\end{array}\right)\left(\begin{array}{c}
n \beta+m-p \\
m-i
\end{array}\right)= \\
=\left(\begin{array}{c}
n \beta \\
p-m
\end{array}\right)(-1)^{m}\left(\begin{array}{c}
n \beta+m-p-n-2 \\
m
\end{array}\right)=\left(\begin{array}{c}
n \beta \\
p-m
\end{array}\right)\left(\begin{array}{c}
-n \beta+n+p+1 \\
m
\end{array}\right), \\
\tilde{V}_{n}^{-1} \tilde{C}_{n} \tilde{D}\left((1+x)^{n \beta}, x\right)^{T} x^{p}=(n+1) !(1-x)^{n-p-1} t_{p}(-n \beta+n+p+1 \mid n \beta, x), \\
\tilde{D}^{-1} \tilde{C}_{n}^{-1} \tilde{V}_{n} x^{p}=\frac{1}{(n+1) !} \sum_{m=p}^{n-1}\left(\begin{array}{c}
n+1+m \\
m
\end{array}\right)^{-1}\left(\begin{array}{c}
n-1-p \\
n-1-m
\end{array}\right) x^{m} .
\end{gathered}
$$

In particular,

$$
\begin{aligned}
T_{n}^{\beta} x^{n-1} & =\left(\begin{array}{c}
2 n \\
n-1
\end{array}\right) \sum_{m=0}^{-1}\left(\begin{array}{c}
n(2-\beta) \\
m
\end{array}\right)\left(\begin{array}{c}
n \beta \\
n-1-m
\end{array}\right) x^{m}, \\
T_{n}^{\beta} x^{0} & =\left(\begin{array}{c}
2 n \\
n-1
\end{array}\right) \sum_{m=0}^{-1}\left(\begin{array}{c}
-n \beta \\
m
\end{array}\right)\left(\begin{array}{c}
n(2+\beta) \\
n-1-m
\end{array}\right) x^{m} .
\end{aligned}
$$

that corresponds to the formula (3).

\section{References}

[1] L. Shapiro, S. Getu, W. Woan, L. Woodson, The Riordan group, Discrete Appl. Math. 34 (1991) 229-339.

[2] R. Sprugnoli, Riordan arrays and combinatorial sums, Discrete Math.132 (1994) 267290.

[3] C. Jean-Louis, A. Nkwanta, Some algebraic structure of the Riordan group, Linear Algebra Appl. 438(2013) 2018-2035.

[4] T.-X. He, L. C. Hsu, P. J.-S. Shiue, The Sheffer group and the Riordan group, Discrete Appl. Math. 155(2007) 1895-1909.

[5] W, Wang, T. Wang, Generalized Riordan arrays, Discrete Math. 308 (2008) 64666500 .

[6] S. M. Roman, The Umbral Calculus, Academic Press, 1984.

[7] V. E. Hoggatt, M. Bicknell, Convolution triangles, Fibonacci Quart. 10 (1972) 599609. 
[8] V. E. Hoggatt, G. E. Bergum, Generalized convolution arrays, Fibonacci Quart. 13 (1975) 193-198.

[9] V. E. Hoggatt, M. Bicknell-Johnson, Numerator polynomial coefficient arrays for Catalan and related sequence convolution triangles, Fibonacci Quart. 15 (1977) 3034 .

[10] G. E. Bergum, V. E. Hoggatt, An application of the characteristic of the generalized Fibonacci sequence, Fibonacci Quart. 15 (1977) 215-220.

[11] V. E. Hoggatt, M. Bicknell-Johnson, Convolution arrays for Jacobsthal and Fibonacci polynomials, Fibonacci Quart. 16 (1978) 385-402.

[12] P. Bala, Notes on generalized Riordan arrays, https://oeis.org/A260492/a260492.pdf

[13] M. Koutras, Eulerian nambers associated with sequences of polynomials, Fibonacci Quart. 32 (1994) 44-57.

[14] B. Drake, An inversion theorem for labeled trees and some limits of areas under lattice paths, A dissertation presented to the Faculty of the Graduate School of Arts and Sciences of Brandeis University, 2008.

[15] P. Bala, Diagonals of triangles with generating function $\exp \left(\mathrm{t}^{*} \mathrm{~F}(\mathrm{x})\right)$, https://oeis.org/A112007/a112007.txt

[16] W. Lang, On generating functions of diagonals sequences of Sheffer and Riordan number triangles, arXiv:1708.01421.

[17] D. V. Kruchinin, V. V. Kruchinin, A generating function for the Euler numbers of the second kind and its application, arXiv:1802.09003.

[18] V. E. Hoggatt, P. S. Bruckman, H-convolution transform, Fibonacci Quart. 13 (1975) 357-368.

[19] E. V. Burlachenko, Riordan arrays and generalized Lagrange series, Math. Notes, 100 (2016) 531-539.

[20] E. Brietzke, An identity of Andrews and a new metod for the Riordan array proof of combinatorial identities, Discrete Math., 308 (2008), 4246-4262.

[21] G.-S. Cheon, S.-T. Jin, Structural properties of Riordan matrices and extending the matrices, Linear Algebra Appl. 435(2011) 2019-2032.

[22] A. Luzon, D. Merlini, M.A. Moron, R. Sprugnoli, Complementary Riordan arrays, Discrete Appl. Math. 172 (2014) 75-87.

[23] P. Bala, Fractional iteration of a series inversion operator, https://oeis.org/A251592/a251592.pdf

[24] P. Bala, A 4-parameter family of embedded Riordan arrays, https://oeis.org/A264772/a264772_1.pdf

[25] S.-L. Yang, Y.-X. Xu, T.-X. He, (m, r)-Central Riordan arrays and their applications, Czechoslovak Math. J. 67(2017) 919-936.

[26] P. Barry, On the halves of a Riordan array and their antecedents, Linear Algebra Appl. 582(2019) 114-137.

[27] T. K. Petersen, Eulerian Numbers, Birkhauser, 2015. 
[28] J. M. Holte, Carries, combinatorics, and an amazing matrix, Am. Math. Mon. 104 (1997) 138-149.

[29] F. Brenti, V. Welker, The Veronese construction for formal power series and graded algebras, Adv. in Appl. Math. 42 (2009) 545-556.

[30] P. Diaconis, J. Fulman, Foulkes characters, Eulerian idempotents, and an amazing matrix, J. Algebr. Comb. 36 (2012) 425-440.

[31] J.-C. Novelli, J.-Y. Thibon, Noncommutative symmetric functions and an amazing matrix, Adv. in Appl. Math. 48 (2012) 528-534.

E-mail: evgeniy_burlachenko@list.ru 